\title{
The extracellular Leucine-Rich Repeat superfamily; a comparative survey and analysis of evolutionary relationships and expression
} patterns

\author{
Jackie Dolan ${ }^{\dagger 1}$, Karen Walshe ${ }^{\dagger 1}$, Samantha Alsbury ${ }^{2}$, Karsten Hokamp ${ }^{1}$, \\ Sean O'Keeffe', Tatsuya Okafuji ${ }^{1}$, Suzanne FC Miller ${ }^{1}$, Guy Tear ${ }^{2}$ and \\ Kevin J Mitchell*1
}

Address: ${ }^{1}$ Smurfit Institute of Genetics, Trinity College Dublin, Dublin 2, Ireland and ${ }^{2}$ MRC Centre for Developmental Neurobiology, New Hunts House, Guys Campus, King's College London SE1 1UL, UK

Email: Jackie Dolan - jadolan@tcd.ie; Karen Walshe - kwalshe@tcd.ie; Samantha Alsbury - samantha.alsbury@kcl.ac.uk;

Karsten Hokamp - kahokamp@tcd.ie; Sean O'Keeffe - limericksean@gmail.com; Tatsuya Okafuji - okafujit@tcd.ie;

Suzanne FC Miller - millers@tcd.ie; Guy Tear - guy.tear@kcl.ac.uk; Kevin J Mitchell* - Kevin.Mitchell@tcd.ie

* Corresponding author †Equal contributors

Published: 14 September 2007

BMC Genomics 2007, 8:320 doi:10.1 |86/|47|-2/64-8-320

This article is available from: http://www.biomedcentral.com/I47I-2I64/8/320

(c) 2007 Dolan et al; licensee BioMed Central Ltd.

This is an Open Access article distributed under the terms of the Creative Commons Attribution License (http://creativecommons.org/licenses/by/2.0), which permits unrestricted use, distribution, and reproduction in any medium, provided the original work is properly cited.
Received: 15 March 2007

Accepted: 14 September 2007

\begin{abstract}
Background: Leucine-rich repeats (LRRs) are highly versatile and evolvable protein-ligand interaction motifs found in a large number of proteins with diverse functions, including innate immunity and nervous system development. Here we catalogue all of the extracellular LRR (eLRR) proteins in worms, flies, mice and humans. We use convergent evidence from several transmembrane-prediction and motif-detection programs, including a customised algorithm, LRRscan, to identify eLRR proteins, and a hierarchical clustering method based on TribeMCL to establish their evolutionary relationships.

Results: This yields a total of 369 proteins (29 in worm, 66 in fly, I 35 in mouse and I 39 in human), many of them of unknown function. We group eLRR proteins into several classes: those with only LRRs, those that cluster with Toll-like receptors (TIrs), those with immunoglobulin or fibronectintype 3 (FN3) domains and those with some other domain. These groups show differential patterns of expansion and diversification across species. Our analyses reveal several clusters of novel genes, including two Elfn genes, encoding transmembrane proteins with eLRRs and an FN3 domain, and six genes encoding transmembrane proteins with eLRRs only (the Elron cluster). Many of these are expressed in discrete patterns in the developing mouse brain, notably in the thalamus and cortex. We have also identified a number of novel fly eLRR proteins with discrete expression in the embryonic nervous system.
\end{abstract}

Conclusion: This study provides the necessary foundation for a systematic analysis of the functions of this class of genes, which are likely to include prominently innate immunity, inflammation and neural development, especially the specification of neuronal connectivity. 


\section{Background}

Leucine-rich repeats (LRRs) are protein-ligand interaction motifs found in a large number of proteins of diverse structure, localization and function in bacteria, fungi, plants and animals [1]. Many of these have well-known functions in the innate immune system [2]. Many others, especially those with extracellular LRRs (eLRRs), are involved in various aspects of nervous system development [3]. In both cases, the nature of the LRR motifs is important for generating a diversity of interactions, with exogenous factors in the immune system and with the huge number of different cell types in the developing nervous system. The structure of LRR motifs and their arrangement in repetitive stretches of variable length generate a versatile and highly evolvable framework for the binding of diverse proteins and non-protein ligands.

Seven classes of LRR have been defined [1]; (these have been referred to as LRR "subfamilies" [4]; we use the term subfamily here in the phylogenetic sense to refer to sets of closely-related genes). Within animals, four separate types are recognised, three typically intracellular and one extracellular. Whether all these different classes are evolutionarily related by descent or represent convergent evolution is open to debate [1] but they all share a characteristic structure. Each repeat is typically 19-29 amino acids long and has a well-conserved N-terminal stretch of 9-12 amino acids that is characterized by precisely-positioned hydrophobic residues (usually leucines) and that forms a $\beta$-strand and a C-terminal stretch of 10-19 amino acids that is more variable in length, sequence and structure. The arrangement of multiple repeats in tandem generates a horseshoe-shaped solenoidal structure, with the $\beta$ strands stacking to form the concave surface and the variable stretches forming the convex surface [1,5-7]. Most LRR regions typically also have both $\mathrm{N}$-terminal and $\mathrm{C}$ terminal cap regions, which shield the hydrophobic core of the LRR structure. In extracellular proteins these regions (LRR-NT and LRR-CT domains, of which several subtypes exist) are defined by precisely positioned cysteine residues [4].

LRR proteins, both intracellular and extracellular, have well-characterized functions in the innate immune system that are similar from plants to mammals [2]. The extracellular LRR (eLRR) proteins in animals include the Toll-like receptors (TLRs), a family of transmembrane proteins characterized by an LRR region, a transmembrane (TM) domain and a cytoplasmic Toll/IL-1 receptor (TIR) domain. This family has expanded in vertebrates to allow detection of a diverse set of antigens [8]. In flies, the TLR family has also expanded, where, in addition to roles in immunity for some of these proteins [9], many are required for various aspects of embryonic and nervous system development [10-13]. Tol-1 in worms is also important in development, possibly contributing to a code of molecules defining neuronal connectivity [14,15]. Recent reports indicate that some mammalian TLR genes may also be expressed and function in neurons $[16,17]$.

A large number of other eLRR proteins have been implicated in various aspects of neural development, genetically in flies [18-20] and in mammals in assays of neurite outgrowth, [21-24], fasciculation [25] and/or synapse formation $[26,27]$. Some of these contain, in addition to the extracellular LRR domain, immunoglobulin (Ig) or fibronectin type-3 (FN3) domains (for review see [3]). In some cases, the functions of eLRR proteins are mediated by homophilic interactions [25,28-30]. In other cases they are mediated by the binding of other proteins in cis [3133] and in trans [27,34-36]. Several eLRR proteins have been found to modulate the signaling of various growth factor pathways (e.g., [37-41]).

Surprisingly, apart from the TLR genes [42] and small secreted proteoglycans [43], relatively few eLRR genes have been studied genetically in mice. Among the ones that have, examples of phenotypic effects in the nervous system include increased plasticity, sprouting and nerve regeneration [44], and defects in axon guidance and cell migration [45], learning and memory [46], myelination $[47,48]$ and neuronal survival [35].

The importance of this class of proteins for nervous system development in humans is apparent from the large number of examples implicated in neurological or psychiatric disorders (reviewed in [49]). These include epilepsy [50], Tourette's syndrome [51], night blindness [52], congenital insensitivity to pain (with mental retardation) [53], and possible links to Alzheimer's disease [54].

Despite the growing number of eLRR proteins implicated in nervous system development or disease this family of proteins has received far less attention as a class than other better characterized families like the immunoglobulin $[55,56]$ and cadherin [57] superfamilies. In particular, there have been no systematic surveys of the genomic complement of these proteins or investigation of their evolutionary relationships. We therefore set out to catalogue the entire extracellular leucine-rich repeat proteome of four organisms: Caenorhabditis elegans, Drosophila melanogaster, Mus musculus and Homo sapiens. We used a hierarchical clustering system to analyse within and betweenspecies relationships, revealing independent diversification and expansion of subfamilies in each species and rapid sequence divergence. These analyses highlight the large number of novel, uncharacterized eLRR proteins in each of these genomes, including several novel subfamilies. A number of these show highly restricted expression in the nervous system in mouse or fly. 


\section{Results \\ Bioinformatics strategy}

We began by obtaining whole proteome sets of known and predicted proteins from each of the four species, as described in Methods. Our initial approach was to filter the starting proteome datasets using transmembrane (TM) and signal peptide prediction programs (TMHMM [58] and SignalP [59]) to identify transmembrane or secreted proteins and then to filter that set using a motif recognition program (Pfam [60]) to identify the subset with motifs of interest. However, this approach using serial filters missed a number of known axon guidance molecules because TMHMM or SignalP misclassified them or Pfam did not detect specific motifs. We therefore included a number of other TM-prediction and motif-recognition programs, including a customised program to look for LRR domains (LRRscan, see below) in the pipeline. In addition, we first performed a clustering step using TribeMCL [61] on the entire proteome sets so as to identify related proteins even where these programs failed to detect specific motifs or architectures. Rather than using any of these programs or a combination of them as strict filters we generated a database containing all the results that could be browsed or searched using various criteria to extract particular gene families of interest (Figure 1).

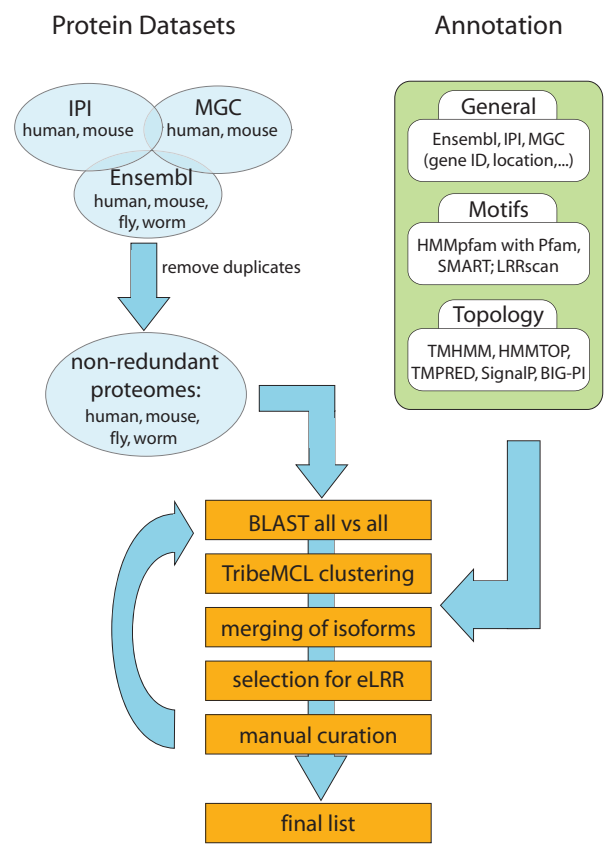

Figure I

Bioinformatics pipeline. Figure shows starting datasets (blue), annotation programs (green) and clustering pipeline (orange) used to generate final eLRR dataset.

\section{Clustering}

TribeMCL is a program designed to cluster proteins into related families based on simultaneous examination of all pairwise BLAST similarity scores [61]. This program uses a Markov cluster algorithm that is particularly well suited to cluster highly divergent proteins with repeated domains into separate subfamilies, a task for which multiple alignment programs are not appropriate. The Markov cluster algorithm is based on simulated 'flow' through a network or graph, where each node is a gene and each connection is weighted by the symmetric pairwise BLAST score. A random walk of a certain length from node to node through this network, which begins within a small cluster of interconnected genes will have a higher probability of ending up within that cluster than crossing to a gene that is only related to one of them. The results of many simulated random walks thus define the clusters. Each time this process is reiterated the links within the clusters that emerge are given a higher weighting and the links that were not used are downgraded. Multiple rounds of this process eventually lead to completely separate clusters. There are two parameters that can be varied that affect the clustering; the first is the e-value cutoff of the BLAST scores that are used, which determines the initial set of connections considered. The second is the inflation parameter; this determines how strongly the links are upgraded with each iteration. A higher inflation parameter increases the 'granularity' of the output; i.e., it generates a larger number of smaller clusters. We used a number of e-value cutoffs (from $\mathrm{e}^{-10}$ to $\mathrm{e}^{-40}$ ) and inflation parameters $(1.2,2,3,4$ and 5) and compared the output produced.

The output from TribeMCL, for any given e-value cutoff and inflation parameter can be viewed as a list of genes organized into clusters with a number assigned to each cluster (lower numbers have more members). We reasoned that hierarchical sorting of genes across various inflation parameters should yield a tree-like structure, with larger clusters at low inflation parameters splitting into more discrete clusters at higher inflation parameters. At each e-value cutoff we therefore sorted the list of genes first at inflation parameter 1.2, then 2, then 3, 4 and 5. For the most part, increasing inflation parameter does lead to splitting of large clusters into smaller clusters and yields a tree-like arrangement of genes with relationships apparent across various levels (but see discussion on "LRR_Tollkin" group below).

\section{Identification of LRR motifs}

To identify LRR proteins, the database was searched for all genes containing at least one LRR, LRR-NT or LRR-CT predicted by either SMART or Pfam. The cutoff values used were based on analysis of the results for proteins with known architecture (see Methods for details). This analysis yielded a total of 2,698 entries. These include both 
genes with intracellular LRRs and those with extracellular LRRs. It also contains isoforms for many genes. To screen out false positives we used the following criteria: if only one LRR was predicted in a gene and only by one of the programs and it was not predicted in either the mouse or human orthologue (for mammalian genes), or in other members of a closely-related cluster then it was considered a false positive and discarded.

\section{Identification of extracellular LRR proteins}

Comparison of several TM-prediction programs suggests that TMHMM is the most reliable, although it is also the most selective [62]. A quick survey of some known TM receptors revealed that TMHMM failed to identify TM domains in several of them, including Robo2 in mammals and Kekkon 2 and 3 in flies, for example. For that reason we also used two other programs, HMMTOP [63] and TMPred [64] to search for TM domains. At least one of these three programs successfully detected the TM domain in all the known TM receptors examined (while also increasing the number of false positives).

SignalP [59] was used to detect signal peptides. This suffered from poor prediction of 5' exons for many mammalian genes, which was solved by manual curation (see below). The GPI-prediction program BIG-PI [65] identified a small number of GPI-linked proteins, including all the known GPI-linked proteins such as Connectin, NgRs and Nyx (the latter in human but not mouse, as reported [66]). A number of other genes were tentatively assigned to the GPI-class by manual inspection based on the presence of a characteristic short C-terminal hydrophobic stretch (and a signal peptide).

In addition to examining the convergent evidence from these various programs to identify eLRR proteins we used three additional criteria. The first is the type of LRR predicted: extracellular proteins typically contain LRR types designated LRR_1，2 or 3 by Pfam or LRR_typical by SMART, while the intracellular proteins have LRR_RI or LRR_sd22 (see [1]). Second, the prediction of an LRR-NT and/or LRR-CT domain was taken as evidence for extracellular localization. Third, especially at low e-value stringencies $\left(e^{-10}\right)$, the majority of extracellular LRR proteins cluster together with TribeMCL in one large group (and a few small ones), distinct from the intracellular proteins. Using these criteria in addition to the data from the prediction programs described above we collected what we believe is a comprehensive set of extracellular LRR proteins across worm, fly, mouse and human. We call these the eLRR proteome.

\section{Manual curation of extracellular sequences}

To reduce the complexity of the final data set a single protein isoform was chosen for each gene and all others were removed (see Methods). Many peptides that we expected to be extracellular because of orthology, clustering or domain structure did not have a predicted signal peptide. Upon manual inspection of the sequences it was discovered that many gene predictions in Ensembl, especially for mammalian genes, were missing the 5'-most exon encoding the signal peptide. For many such genes we identified the $5^{\prime}$ exon and the full coding sequence in a sequence from another database and/or by searching with an orthologous gene from mouse or human. In other cases the 5 ' predicted sequence extended past the apparent true methionine start codon, which could be recognised by conservation and the presence of the signal sequence.

We identified two fly genes that have been incorrectly annotated in Ensembl as two separate genes each. CG32637 and CG4187 represent the 5' and 3' ends of a cDNA encoded by AB134171, a new member of the Lgr3 family. Similarly, CG4054 and CG13487 represent the 5' and $3^{\prime}$ ends of the fish-lips (fili) gene, encoded by AAV36870 [67] which is related to tartan and capricious $[67,68]$. We detected one similar mis-annotation in the worm database (pxn-1) and presume that this type of error may also have occurred for some mammalian sequences.

All the manually curated gene sequences are provided [see Additional File 1]. These curated sequences were fed back into the starting database and the BLAST and clustering analyses were re-performed to ensure that spurious results had not been generated by incorrect sequences.

\section{Defining consensus architectures}

In order to derive a consensus architecture for each gene we compared the results of SMART and Pfam and the TMprediction programs. Even at very low stringency some LRRs in proteins with known numbers of such repeats were missed by HMMpfam using the SMART and Pfam databases. This includes a number of somewhat degenerate LRRs in Lrrc8 proteins [69], for example, as well as atypical LRR-CT domains in small proteoglycans and Gprotein-coupled receptors [4]. For this reason, using a similar strategy to Smits and colleagues [69], we wrote a customised program, LRRscan, to search for a more inclusive minimal consensus that defines LRRs as well as searching for consensus sequences derived from noncanonical LRR-CT domains (see Methods for details). LRRscan was successful in identifying all the predicted LRRs in Lrrc8 proteins, including atypical or degenerate ones [69], and additional LRRs in many other proteins that were not detected by HMMpfam with SMART or Pfam.

The output from LRRscan and HMMpfam was compared for all proteins [see Additional File 2] and a consensus 
architecture including number of LRRs and presence of LRR-NT and LRR-CT domains was derived by manual curation. The consensus matches the architecture of a number of eLRR proteins with published structures [57,70-72], allowing for semantic differences in how the LRRs are counted. The final LRR before the LRR-CT domain (CT1 subtype) often contains only the first subdomain of nine residues; following the convention of Matsushima and colleagues [49] we count this as one repeat rather than part of the LRR-CT domain, which in some cases may cause an apparent discrepancy with published reports. We also do not count in the total number of repeats putative LRRs which overlap with LRR-NT or LRR-CT domains, as has been done in some published cases $[73,74]$.

A consensus topology for each protein was also derived by comparison of the signal peptide, GPI anchor and TMprediction programs. A full list of all the eLRR proteins is provided [see Additional File 3] and a sample is shown in Figure 2. These are sorted hierarchically across inflation parameters at an e-value cutoff of -40 . Clustering results at e- 10 and e- 25 are also presented [see Additional Files 4 and 5]. Figures 3 and 4 provide an overview of the consensus protein architectures of most of the eLRR proteins, arranged in subfamilies. A large number of singleton LRR_Only proteins are not shown in this diagram (these are listed separately in Table 1 ).

\section{The eLRR superfamily}

We categorized the eLRR proteins into four classes, based on their architecture and clustering. These are LRR_Ig/Fn3 (containing an Ig or FN3 domain but no other extracellular domains except LRRs), LRR_Tollkin (containing a cytoplasmic TIR domain or clustering with the Toll proteins), LRR_Other (containing some other domain, such as EGF repeats or a G-protein-coupled receptor domain) and LRR_Only (containing no other recognizable domain). These categories are broadly supported by the clustering results, although the LRR_Other group is clearly arbitrary and contains a number of unrelated subfamilies. The number of eLRR proteins in each of these classes in each of the four organisms studied is shown in Table 2. These are broken down into several categories, based on predicted localization: secreted, GPI-linked, type I transmembrane and multi-membrane spanning (all multimembrane spanning proteins were classified into the LRR_Other group). Almost all of the LRR_Ig/FN3 group are associated with the plasma membrane, either as type I TM or GPI-linked proteins. In contrast, the LRR_Tollkin and LRR_Only groups contain a far higher percentage of secreted proteins. It is clear from an examination of these data that the eLRR superfamily has greatly expanded in mammals (>135 genes) and to a lesser extent, flies (66), compared to worms (29).

\section{Subfamily expansion and diversification}

In order to assess the extent of expansion (new members of existing subfamilies) and diversification (new subfamilies) across different organisms, we analysed the membership of clusters across mouse and fly. For this purpose, we defined clusters in such a way as to distinguish those with species-specific expansion from those with diversification [see Additional File 6]. For each cluster we counted the number of fly and mouse members and then generated histograms of the number of clusters with $\mathrm{x}$ fly members and y mouse members (Figure 5). For example, in the LRR_Ig/FN3 group there is one cluster with one fly gene and three mouse genes (Lrigs) and there are six clusters with no fly genes and three mouse genes (Ntrk, Lrrn13, Lrrc4, Amigo, FLRT and Lrrc21 groups). These graphs illustrate the different rates of expansion and diversification across these groups.

For the LRR_Ig-FN3 family there is a large number of clusters that have multiple mouse genes and no fly genes. These represent the diversification of new architectures and gene families in the mammalian lineage. There is only one case of expansion in the mouse within a conserved subfamily (the Lrig family which has three members in mouse and one, lambik, in fly (as well as one in worm)). Conversely, the kekkon family shows a specific expansion in flies compared to mammals, where there is only a single apparent closest orthologue, Lrrc24.

In contrast, in the LRR_Tollkin group there has been independent expansion of subfamilies in both flies and mammals (and even comparing mouse and human). Similar expansions are observed in the subfamilies of Toll-like receptor genes themselves and in the subfamilies of gene encoding proteins that do not have TIR domains but that cluster within this group (see below).

In the LRR_Only group there has also been independent expansion, apparently followed by rapid divergence, resulting in a very large number of singletons in each species. These are genes with no recognizable orthologue in the other species (fly or mouse) and no recognizable paralogue in their own species. The encoded proteins do not cluster at high stringency (e-value and inflation parameter) but many cluster into a very large group at lower stringency. This trend may reflect increased divergence rates of this class of proteins. There is only one case in this group of apparent orthology, between CG6959 in fly and Tpbg/ 5T4 [75] and a novel gene in mouse.

The LRR_Other group shows the opposite pattern with the largest number of clear orthologues between mouse and fly (clusters on the diagonal). This group also contains the most clusters with a clear worm orthologue [see Additional File 3 and Figure 4]. The members of this group 


\begin{tabular}{|c|c|c|c|c|c|c|c|}
\hline External_Name & Synonyms & clust1_2 & clust2 & clust3 & clust4 & clust5 & Location \\
\hline Lrig1 & & 156 & 1728 & 1685 & 1622 & 1591 & 6:95066418-95161799 \\
\hline Lrig2 & & 156 & 1728 & 1685 & 1622 & 1591 & 3:103884357-103939517 \\
\hline Lrig3 & & 156 & 1728 & 1685 & 1622 & 1591 & $10: 125665573-125714974$ \\
\hline lbk & Lambik & 156 & 1728 & 1685 & 1622 & 1591 & 2R:11679210-11684383 \\
\hline T21D12.9 & & 156 & 1728 & 1685 & 1622 & 1591 & IV: $274570-287201$ \\
\hline Lrrn6a & Lingo 1 & 636 & 1937 & 1898 & 1838 & 1809 & $9: 56731965-56798743$ \\
\hline Lrrn6b & Lingo3 & 636 & 1937 & 1898 & 1838 & 1809 & $10: 80964866-80976093$ \\
\hline Lrrn6c & Lingo2 & 636 & 1937 & 1898 & 1838 & 1809 & $4: 35846472-36195578$ \\
\hline Lrrn6d & Lingo4 & 636 & 1937 & 1898 & 1838 & 1809 & 3:93887043-93892325 \\
\hline Lrrn1 & Nlrr1 & 636 & 3698 & 3710 & 3680 & 3664 & $6: 108031528-108072472$ \\
\hline Lrrn2 & Nlrr2 & 636 & 3698 & 3710 & 3680 & 3664 & $1: 132741816-132801420$ \\
\hline Lrrn3 & Nlrr3 & 636 & 3698 & 3710 & 3680 & 3664 & $12: 38061168-38095273$ \\
\hline Lrrc4 & Ngl-2 & 636 & 5118 & 5199 & 5214 & 5224 & $6: 28876652-28878610$ \\
\hline Lrrc $4 b$ & Ngl-3 & 636 & 5118 & 5199 & 5214 & 5224 & $7: 38517725-38540037$ \\
\hline $\operatorname{Lrrc4c}$ & Ngl-1 & 636 & 5118 & 5199 & 5214 & 5224 & 2:97172341-97336348 \\
\hline XP_485967 & Garp/ Lrrc32 & 671 & 2804 & 5902 & 5974 & 5994 & unmapped \\
\hline Lrrc33 & Garpll & 671 & 2804 & 7897 & 8069 & 8147 & $16: 30952197-30974848$ \\
\hline Lrrc 15 & Lib & 671 & 3320 & 9586 & 9863 & 10054 & $16: 29076635-29091261$ \\
\hline Gp5 & Glycoprotein 5/GPV & 671 & 3320 & 10192 & 10520 & 10743 & $16: 29115637-29118723$ \\
\hline Cpn2 & Carboxypeptidase N, polypeptide 2 & 671 & 3320 & 12102 & 12561 & 12887 & 16:29063712-29074853 \\
\hline Igfals & Als & 671 & 7252 & 7532 & 7676 & 7734 & $17: 24607540-24609348$ \\
\hline 1200009O22Rik & mKIAA0644 & 671 & 10234 & 10860 & 11238 & 11494 & 6:53745044-53750272. \\
\hline CG7509-PA & & 671 & 25631 & 27360 & 28472 & 29304 & 3L:4783924-4786121 \\
\hline C56E6.6 & & 1796 & 5143 & 8386 & 22037 & 22689 & II:6540165-6544297 \\
\hline CG40500-PD & & 1796 & 5143 & 8386 & 11883 & 14994 & Xh:214902-235557. \\
\hline CG5195-PA & & 1796 & 5143 & 8386 & 27788 & 28588 & 3L:20388531-20398189 \\
\hline CG7896-PA & & 1796 & 5143 & 8386 & 11883 & 29432 & 3R:25809174-25816514 \\
\hline $\operatorname{chp}$ & Chaoptin & 1796 & 5143 & 13751 & 25152 & 25868 & 3R:27029904-27036452 \\
\hline CG4168-PA & & 1796 & 5143 & 13751 & 27464 & 28254 & 2L:15158036-15174249 \\
\hline CG8561-PA & & 1796 & 5143 & 27662 & 28794 & 29638 & 2R:9786463-9789735 \\
\hline Tol-1 & & 1796 & 7985 & 8352 & 8558 & 8674 & I:444002-461590 \\
\hline Toll-7 & dToll7 & 1796 & 7985 & 8352 & 8558 & 8674 & 2R:15341768-15346108 \\
\hline $18 w$ & 18 Wheeler & 1796 & 7985 & 8352 & 8558 & 8674 & 2R:15626374-15631795 \\
\hline Tollo & dToll8 & 1796 & 7985 & 8352 & 8558 & 8674 & 3L:15200900-15208091 \\
\hline Toll-6 & dToll6 & 1796 & 7985 & 8352 & 8558 & 8674 & 3L:15301959-15307470 \\
\hline $\mathrm{Tl}$ & dToll1 & 1796 & 9274 & 9759 & 10055 & 12016 & $3 R: 22624765-22668125$ \\
\hline MstProx & dToll3 & 1796 & 9274 & 9759 & 10055 & 12016 & 3R:3191661-3195027 \\
\hline Toll-4 & dToll4 & 1796 & 9274 & 9759 & 10055 & 12016 & 2L:9084107-9089440 \\
\hline Tehao & dToll5 & 1796 & 9274 & 9759 & 10055 & 29162 & 2L:13435637-13440955 \\
\hline Chad & Chondroadherin & 4838 & 5233 & 11926 & 12369 & 12678 & $11: 94386155-94390215$ \\
\hline XP_001001535 & & 4838 & 5233 & 8540 & 8733 & 8858 & $15: 81522436-81523284$ \\
\hline
\end{tabular}

\section{Figure 2}

Sample from list of all eLRR genes, hierarchically clustered at $\mathrm{e}^{-40}$ cutoff. Proteins have been sorted in this table based on the clustering output from TribeMCL. This has been done hierarchically across inflation parameters, starting at I.2, then 2, 3, 4 and 5. For most proteins this yields a tree-like structure with cluster stringency increasing (and membership decreasing) from low inflation parameters to high. Numbers used to identify clusters are generated by TribeMCL with larger clusters having lower numbers. Proteins are colour-coded by species: black, mammalian; blue, fly; red, worm. For the mammalian proteins, only the mouse orthologue is listed. The table shows examples of clusters in the LRR_Ig/FN3 group with mouse, fly and worm orthologues (the Lrig subfamily) and with mouse paralogues only (the Lrrn6, Lrrn I-3 and Lrrc4 subfamilies, which cluster together at level I.2). It also shows many of the proteins in the LRR_Tollkin group, with the hierarchical clustering apparent across inflation parameters and indicated by shading. One subfamily containing a known and novel member is shown at the bottom. Proteins encoded by genes located in tandem in the genome are boxed in the right-hand column. A complete list of all eLRR proteins is provided [see Additional File 3]. Lists clustered at the $\mathrm{e}^{-25}$ and $\mathrm{e}^{-10}$ cutoff levels are given [see Additional Files 4 and 5]. 
LRR_Ig/FN3 group
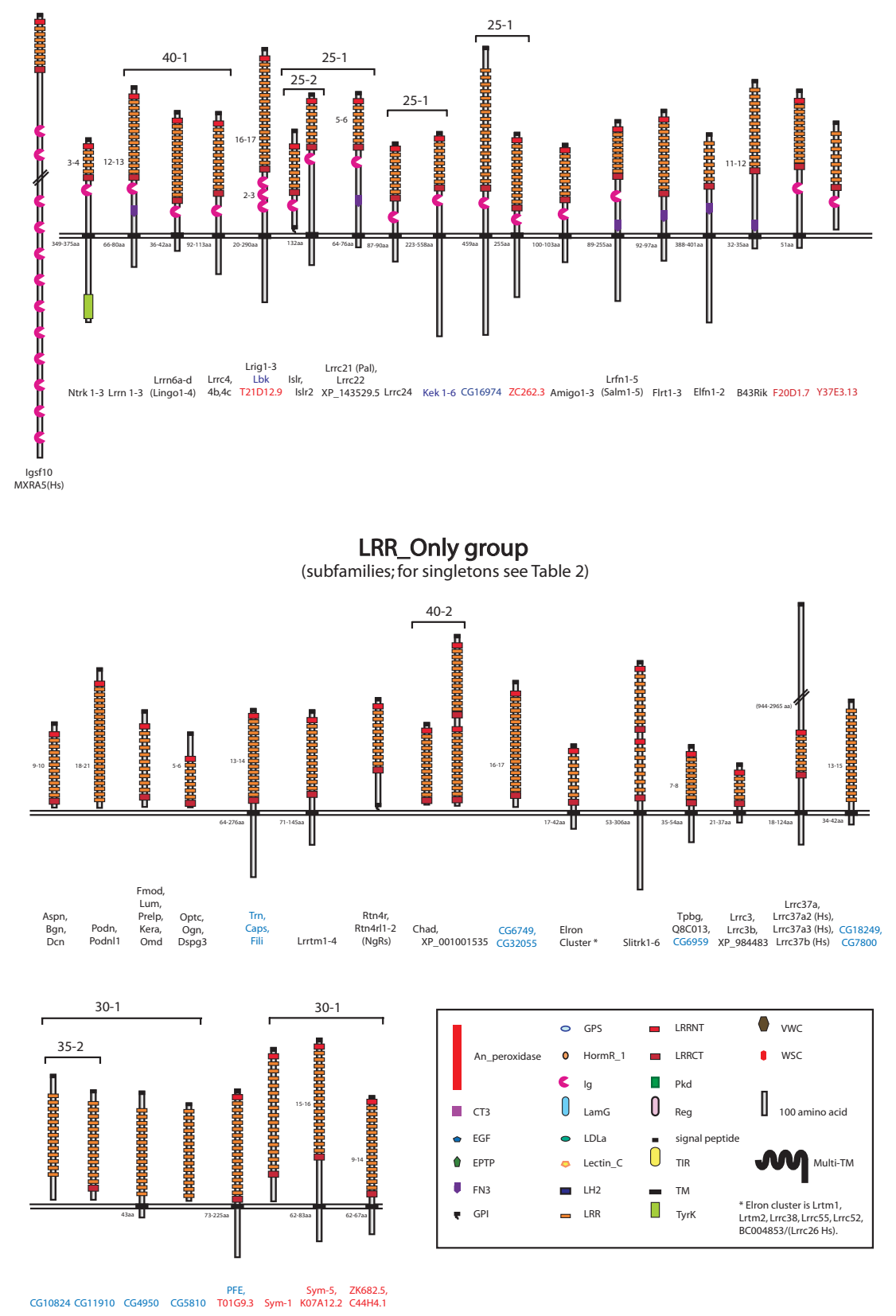

Figure 3

eLRR protein predicted architectures (part I). Consensus architectures are shown for all proteins in the LRR_Ig/FN3 group and for all proteins in subfamilies in the LRR_Only group. An additional set of LRR_Only singletons is listed separately in Table I. Protein names are shown below the corresponding structures (black, mammalian; blue, fly; red, worm). All figures are drawn to scale (see Key). Consensus architectures were derived for single proteins and across subfamilies from convergent evidence from motif and topology prediction programmes. Where there is a range in number of predicted LRRs or other domains across members of a subfamily, this is indicated next to the domain. A range in length of the cytoplasmic domain is similarly indicated, where it exceeds 20 amino acids. Tightly clustered subfamilies (e.g., Slits, Amigos) are listed under a single consensus architecture. Clusters with more structurally diverse proteins are indicated by the brackets; the numbers refer to evalue and inflation parameter at which the proteins cluster in the MCL programme. See Key for more information. 


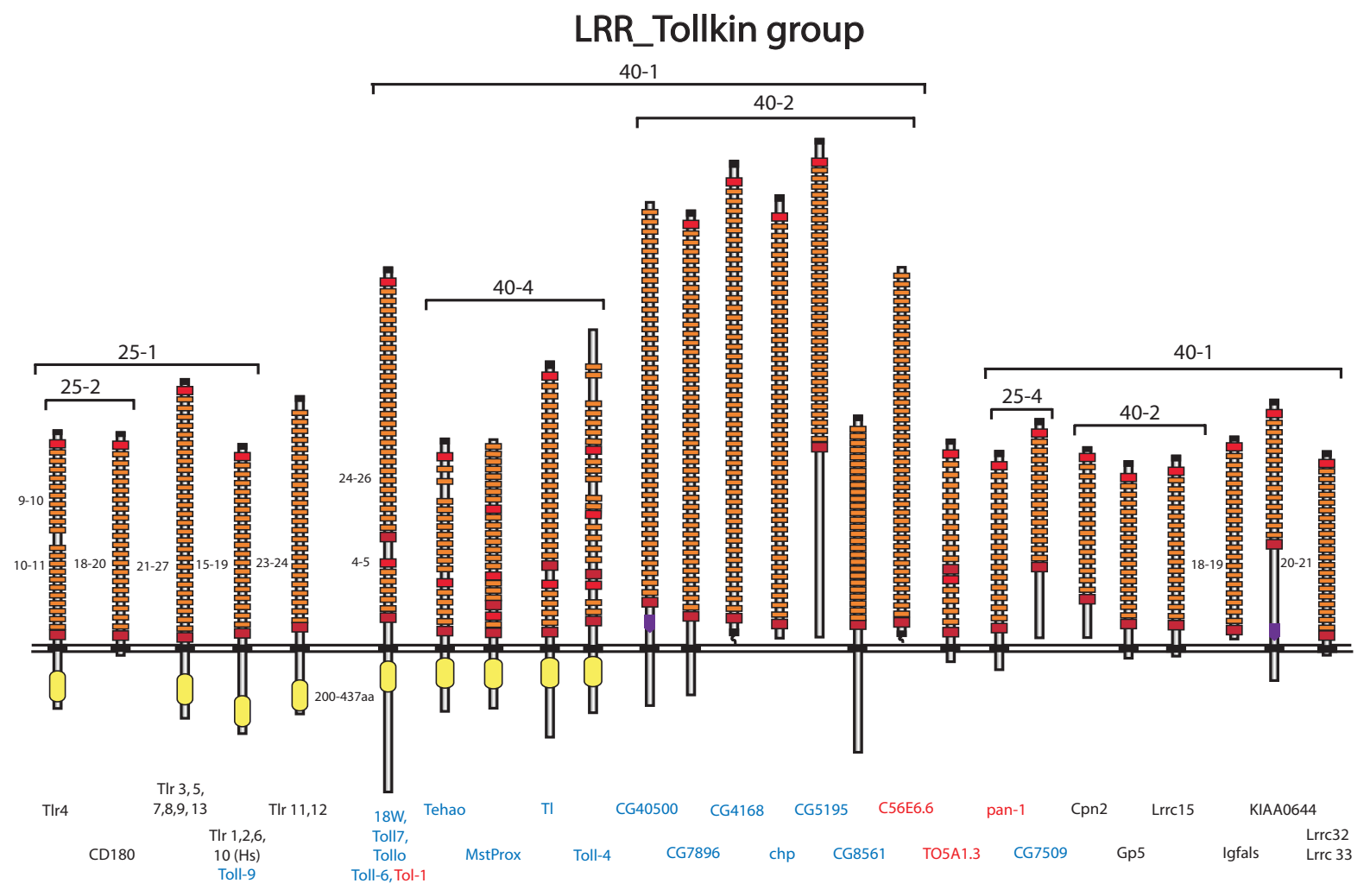

\section{LRR_Other Group}

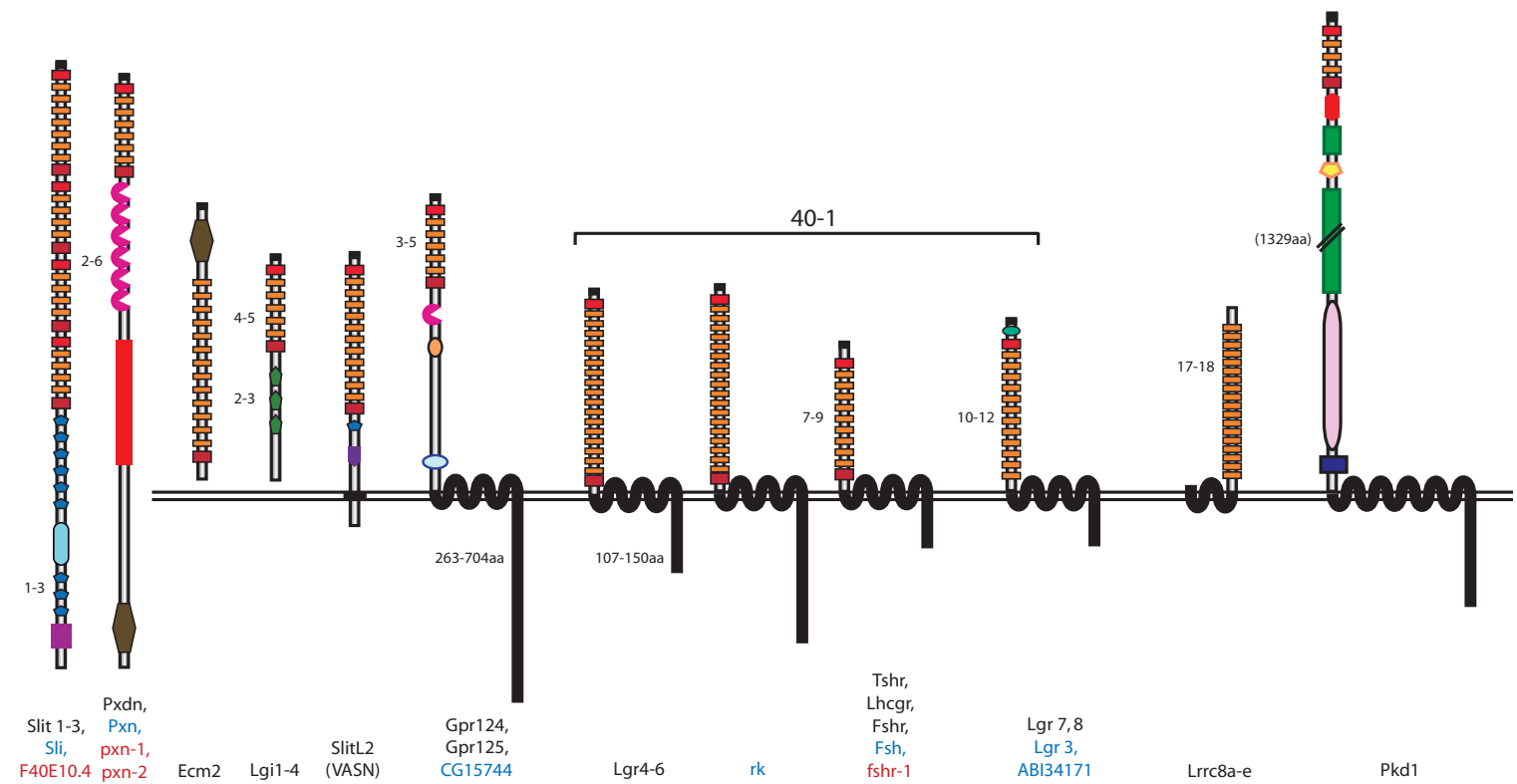

Figure 4

eLRR protein predicted architectures (part 2). Consensus architectures are shown for all proteins in the LRR_Tollkin and LRR_Other groups. See Figure 3 legend for details. 
Table I: List of LRR_Only singletons

\begin{tabular}{|c|c|c|c|}
\hline Symbol & Name/synonyms & Peptide length & Consensus architecture \\
\hline \multicolumn{4}{|c|}{ Mammalian proteins } \\
\hline $\mathrm{BC} 031901$ & novel & 872 & SS, 7LRR, TM \\
\hline CdI4 & & 366 & SS, LRRNT, IILRR, GPI \\
\hline Gplba & Glycoprotein Ib, alpha polypeptide & 734 & SS, LRRNT, 8LRR, LRRCTI, TM \\
\hline Gplbb & Glycoprotein Ib, beta polypeptide & 214 & SS, LRRNT, 2LRR, LRRCTI, TM \\
\hline Gp9 & Glycoprotein 9 & 177 & SS, LRRNT, 2LRR, LRRCTI, TM \\
\hline Lrgl & Leucine-rich alpha-2-glycoprotein I & 342 & SS, LRRNT, 9LRR, LRRCT2 \\
\hline Lrrcl7 & & 443 & SS, LRRNT, 4LRR, LRRCTI, LRRNT, 3LRR, LRRCTI \\
\hline Lrrcl9 & & 364 & SS, LRRNT, 6LRR, LRRCTI, TM \\
\hline Lrrc25 & & 297 & SS, 2LRR, LRRCTI, TM \\
\hline Nepn & Nephrocan/573052 IEI2Rik & 512 & SS, LRRNT, I7LRR, LRRCTI \\
\hline Nyx & Nyctalopin (mouse) & 476 & SS, LRRNT, IILRR, LRRRCTI, TM \\
\hline NYX & Nyctalopin (human) & 481 & SS, LRRNT, I2LRR, LRRRCTI, GPI \\
\hline Omg & Oligodendrocyte myelin protein & 440 & SS, LRRNT, 7LRR, LRRCT2, GPI \\
\hline Q7Z2Q7 & Synleurin (human) & 621 & SS, LRRNT, I 3LRR, LRRCTI, TM \\
\hline Tsku & Tsukushi/Lrrc54 & 354 & SS, LRRNT, IOLRR, LRRCT2 \\
\hline \multicolumn{4}{|c|}{ Fly proteins } \\
\hline Con & Connectin & 691 & SS, LRRNT, I ILRR, LRRCTI, GPI \\
\hline GpI50 & GpI50 & 1051 & SS, LRRNT, I5LRR, LRRCT2, TM \\
\hline hfw & Halfway & 611 & SS, LRRNT, 4LRR, LRRNT, 2LRR, LRRCTI \\
\hline$w d p$ & windpipe & 677 & SS, LRRNT, 4LRR, LRRCTI, TM \\
\hline CGI504 & & 392 & IILRR, LRRCTI, TM \\
\hline CG478I & & 469 & SS, LRRNT, I ILRR, LRRCTI, TM \\
\hline CG5096 & & 491 & SS, LRRNT, I2LRR, LRRCT, TM \\
\hline CG554I & & 463 & SS, LRRNT, 6LRR, TM \\
\hline CG5819 & & 915 & SS, LRRNT, I7LRR, LRRCTI, TM \\
\hline CG5888 & & 455 & SS, LRRNT, 8LRR \\
\hline CG7702 & & 537 & SS, LRRNT, I ILRR, LRRCTI, TM \\
\hline CG8852 & & 663 & SS, IOLRR, LRRCT, TM \\
\hline CGI0I48 & & 329 & SS, 9LRR \\
\hline CGIII36 & & 799 & SS, LRRNT, I 3LRR, LRRCTI, TM \\
\hline CGI435I & & 1316 & SS, LRRNT, I2LRR, LRRCTI, TM \\
\hline CGI4662 & & 550 & SS, 6LRR, TM \\
\hline CGI4762 & & 470 & SS, LRRNT, I4LRR, LRRCTI \\
\hline CGI5658 & & 343 & SS, LRRNT, 7LRR, LRRCTI, TM \\
\hline CGI7667 & & 458 & SS, LRRNT, 7LRR, TM \\
\hline CGI8095 & & 548 & SS, I8LRR, TM \\
\hline CGI8480 & & 550 & SS, LRRNT, 7LRR, LRRCT, TM \\
\hline CG32372 & & 817 & SS, 23LRR \\
\hline \multicolumn{4}{|c|}{ Worm proteins } \\
\hline $\mathrm{C} 02 \mathrm{C} 6.3$ & & 369 & SS, LRRNT, 8LRR, LRRCTI, GPI \\
\hline C4IC4.3 & & 630 & SS, 8LRR \\
\hline FIOF2.4 & & 656 & SS, LRRNT, I8LRR, LRRCTI, TM \\
\hline F37E3.2 & & 568 & SS, LRRNT, I ILRR, TM \\
\hline K03AI.2 & & 586 & SS, LRRNT, 9LRR, LRRCTI, TM \\
\hline T22E7.Ia & & 341 & SS, 8LRR, LRRCTI, TM \\
\hline T23GII.6 & & 653 & SS, LRRNT, I5LRR, LRRCT, TM \\
\hline Y39AIA.7 & & 187 & SS, LRRNT, 4LRR \\
\hline Y7IF9B.8 & & 542 & SS, LRRNT, I4LRR, LRRCTI, TM \\
\hline Y75B8A.5 & & 448 & SS, LRRNT, 6LRR, LRRCTI \\
\hline Y76A2B.2 & & 782 & SS, LRRNT, 6LRR, GPI \\
\hline
\end{tabular}

List of singleton proteins in LRR_Only group not shown in Figure 3. For the mammalian proteins, only the mouse orthologue is listed, with the following exceptions: both human and mouse Nyctalopin ( Nyx) are listed as they have different topologies (GPI-linked and TM, respectively) and synleurin is a human gene that has been pseudogenised in mouse. 
Table 2: Complement of eLRR proteins by group, localisation and species

\begin{tabular}{llllll}
\hline LRR_Ig/FN3 & & & & \\
& Type I TM & GPI & Secreted & Multi-TM & Total \\
\hline Worm & 3 & 0 & 1 & 0 & 4 \\
Fly & 8 & 0 & 0 & 0 & 8 \\
Mouse & 35 & 1 & 1 & 0 & 37 \\
Human & 35 & 1 & 2 & 0 & 38 \\
Total & 81 & 2 & 4 & 0 & 87
\end{tabular}

\section{LRR_Tollkin}

\begin{tabular}{llllll}
\hline Worm & 3 & 1 & 0 & 0 & 4 \\
Fly & 12 & 1 & 3 & 0 & 16 \\
Mouse & 17 & 0 & 2 & 0 & 19 \\
Human & 17 & 0 & 2 & 0 & 19 \\
Total & 49 & 2 & 7 & 0 & 58 \\
\hline
\end{tabular}

LRR_Other

\begin{tabular}{llllll}
\hline Worm & 0 & 0 & 3 & 1 & 5 \\
Fly & 0 & 0 & 2 & 5 & 16 \\
Mouse & 1 & 0 & 9 & 26 \\
Human & 1 & 0 & 23 & 16 & 38 \\
Total & 2 & 0 & 63 & \\
\hline
\end{tabular}

\section{LRR_Only}

\begin{tabular}{llllll}
\hline Worm & II & 2 & 4 & 0 & 17 \\
Fly & 23 & 1 & 10 & 0 & $35^{*}$ \\
Mouse & 28 & 5 & 19 & 0 & 52 \\
Human & 32 & 6 & 19 & 57 \\
Total & 94 & 14 & 52 & 0 & $16 I^{*}$ \\
\hline
\end{tabular}

The numbers of eLRR proteins in each of the four major groups is listed for each species, broken down by predicted protein localisation or topology: type I transmembrane, GPI-linked, secreted and multiple-membrane-spanning. *includes CG I 504, unclassified localisation.

include the Slit proteins, peroxidasins, and a number of G-protein coupled hormone receptors, which are all conserved, as well as a number of mammal-specific families including the Lgi proteins.

\section{Clustering of known proteins}

These analyses provide an overview of relationships within the eLRR superfamily and highlight a number of previously unreported associations, allowing us to classify several novel proteins as paralogues of Lrrc21/Pal, Tpbg/ 5T4, Lrrc3 or Chad, for example. Conversely, it is clear that the recently named NLRR4 is not in fact a paralogue of the other NLRR proteins (-1,2 and 3; also confusingly known as Lrrn 1, 2 and 3). Also, the Lrig proteins in mammals are orthologous not to kekkon proteins in the fly, as has been suggested previously [76], but to the lambik protein in flies (and T21D12.9 in worms). The mammalian protein Lrrc24 appears to be the closest orthologue of the kekkon proteins.
A particularly interesting finding is of a number of LRR proteins which cluster with the Toll-like receptors in both flies and mammals but which do not have a characteristic TIR domain. One of these: CD180, also known as RP105, clusters specifically with Tlr4. This protein lacks a TIR domain and has recently been found to act as a negative regulator of Tlr4 [77]. Also in the LRR_Tollkin group in mammals is a subgroup of more distantly related proteins: carboxypeptidase $\mathrm{N}$ subunit 2 (Cpn2), glycoprotein $\mathrm{V}$ (Gp5) and leucine-rich repeat-containing protein 15 (Lrrc15, also known as Lib), (which form a sub-cluster), as well as insulin-growth factor acid labile subunit (Igfals) and KIAA0644 (which also has an FN3 domain). Lrrc32 (also known as GARP [78]) and the related protein Lrrc33 also fall into this cluster, along with the novel fly protein CG7509. In the fly there is also another subcluster that clusters with the Tlrs. This subcluster includes chaoptin, which is known to function as an adhesion molecule in neural development [18] and several other novel proteins, 

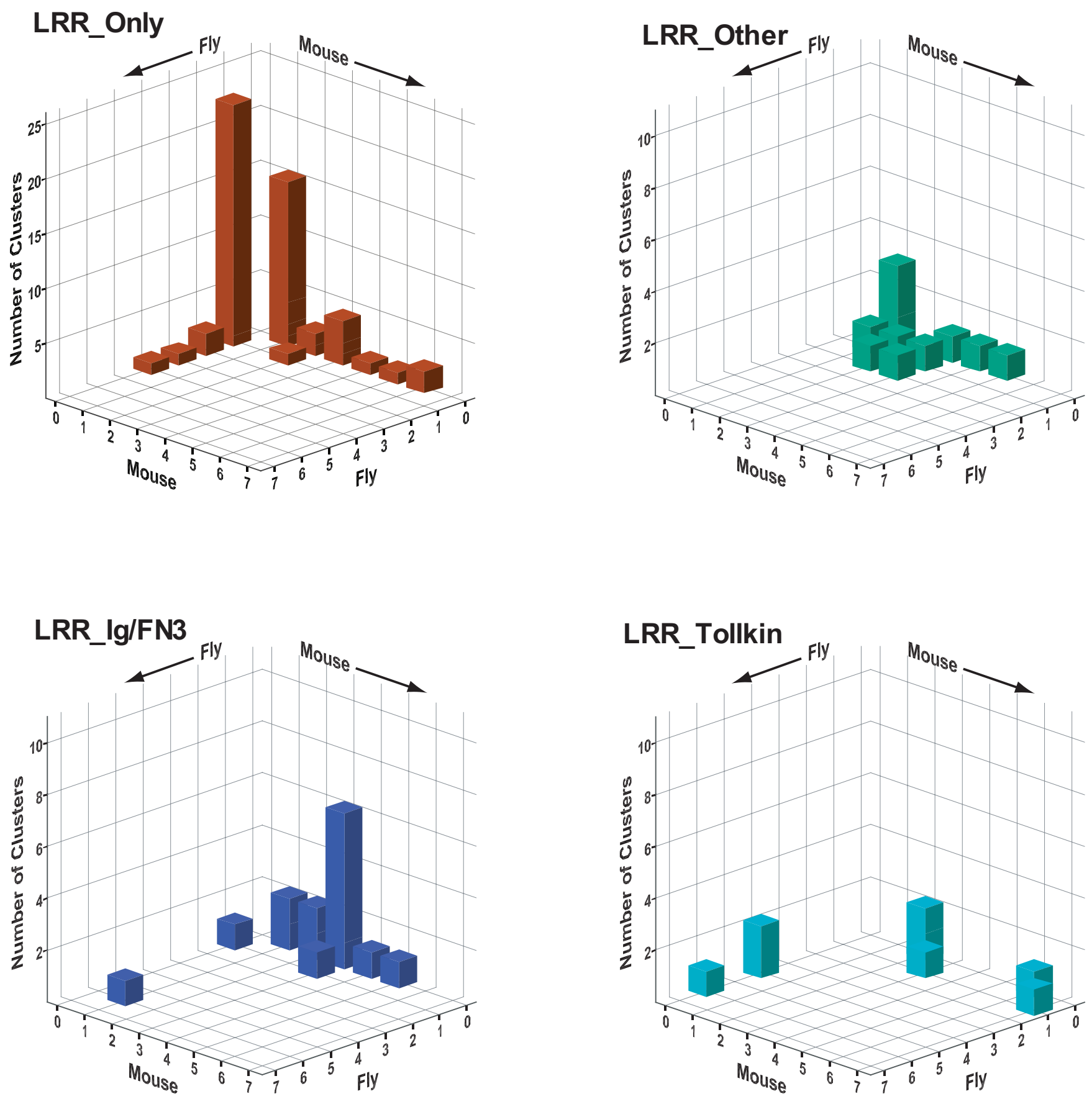

Figure 5

Group-specific patterns of expansion and diversification. The graphs depict three-dimensional histograms showing the number of clusters (on the $z$ axis) having $x$ members in the fly and $y$ members in the mouse. The clusters used for this analysis are listed [see Additional File 6]. Different patterns of expansion (new members in one species of a conserved subfamily) and diversification (novel subfamilies in one species) are observed across the four major groups of eLRR proteins. Graphs were generated with the SPSS program.

including one with an FN3 domain (CG40500-PD). The chaoptin cluster also contains the worm tol-1 protein and the novel worm protein C56E6.6. The large LRR_Tollkin group is one example where the expectation of hierarchi- cal clustering does not hold; in many cases, individual proteins in this broad family cluster into different subfamilies at different e-values and inflation parameters [see Additional File 4 and discussion]. 


\section{Novel protein families}

These analyses have also catalogued a large number of novel proteins and subfamilies encoding eLRR proteins in worms, flies and mammals. Two novel clusters in mammals are of special interest due to their expression patterns in the nervous system (see below). One includes two closely related TM proteins currently identified as A930017N06Rik and Lrrc62 in the mouse. These proteins form a distinct subfamily at high stringency and are characterized by a signal peptide, 6 LRR repeats, an LRR-CT and an FN3 domain extracellularly, a TM domain and a long cytoplasmic tail (Figures 3, 6). The cytoplasmic tail contains a large number of tyrosines but no other detectable motifs. Both genes have two exons with the coding sequence entirely in the 3' exon. We propose to name these Elfn proteins, for extracellular-Leucine-rich repeat Fibronectin domain proteins. (A930017N06Rik is Elfn1 and Lrrc62 is Elfn2).

Another cluster of related proteins comprises BC004853 (called LRRC26 in humans), Lrrc38, Lrrc52 and Lrrc55, Lrtm1 and Lrtm2 (names derived from sequencing projects [79]). These are all LRR_Only proteins with a signal peptide, an LRR-NT, 6 LRR repeats, an LRR-CT, a TM domain and a short cytoplasmic tail containing a short stretch of acidic residues (Figures 3, 7). Lrtm 1 and 2 also contain conserved predicted PDZ-binding sequences at their C-termini, suggestive of synaptic localisation. These proteins cluster in a group of six at low stringency $\left(e^{-25}\right.$, level 1), but break into several subclusters at higher stringency (including Lrtm 1 and 2 and Lrrc38 and 55). They are defined as paralogues in the Ensembl database but whether they represent a true "subfamily" is unclear. For convenience, we refer to this cluster as the "Elron" cluster, for extracellular-Leucine-Rich repeat-Only proteins, but have not renamed individual members.

In flies there are several subfamilies of novel proteins. These include CG7800 and CG18249, both LRR_Only TM proteins, CG32055 and CG6749, both secreted LRR_Only proteins and CG10824 and CG11910, which cluster as a pair at $e^{-35}$, level 2 and in a group of four with CG4950 and CG5810 at $e^{-25}$, level 1. CG4950 is a predicted TM protein while the others in this cluster are predicted secreted proteins.

In worms, a subfamily emerges comprising sym-1 (C44H4.3) and sym-5 (C44H4.2), both of which interact genetically with mec-8[80], along with $\mathrm{C} 44 \mathrm{H} 4.1$ and two other predicted proteins K07A12.2 and ZK682.5. There are also several cases of apparent one-to-one worm-fly orthology of novel proteins, including CG16974 and ZC262.3a, CG7509 and M88.6a, CG15151 and T01G9.3 and CG5819 and K07A12.2.

\section{Genomic clustering}

To assess the possibility that some related genes might occur in clusters in the genome we examined genomic locations for all genes in our eLRR dataset [see Figure 2 and Additional File 3]. Not surprisingly, many closely related genes occur in tandem: five of the six Slitrk genes occur in two clusters in the mouse, one on the X chromosome and one on chromosome 14 . Other genes occurring in tandem include Tlr7 and Tlr8, Islr1 and Islr2, Lrrc21/Pal and Lrrc22, Lrrc8b, $c$ and $d$ and Fshr and Lhcgr. We also found a number of examples where more distantly related genes occur in tandem in the genome, lending further support to the clustering results presented above, including Cpn2, Gp5 and Lrrc15/Lib. In the fly, several Tollrelated genes occur in adjacent pairs (Tollo with Toll-6, Toll-7 with $18 w$ and Toll-9 with CG5195, a novel non-TIRcontaining member of the LRR_Tollkin group), as do tartan and capricious. Similarly, a number of the novel subfamilies identified above occur in tandem including CG7800 with CG18249, CG32055 with CG6749 and CG10824 with CG5810. In the worm genome, the C44H4 genes $(.1, .2(s y m-5)$ and $.3(s y m-1))$ also occur in tandem.

We observed an interesting situation in the family of small secreted proteoglycans that includes decorin, biglycan, and related genes. Proteins in this family fall into several subclusters using TribeMCL, in agreement with previous analyses [81]. Interestingly, many of them are also grouped in tandem in the genome in several different loci but each locus contains a representative of two or three subclusters. This suggests two early duplications in tandem and a subsequent triplication of the entire locus, with some additional gene losses and duplications [82]. The Ecm2 gene is also located in tandem in one of these loci (with Aspn, Omd and Ogn) but it is highly divergent from the other proteins and whether it should be considered a member of this family is debatable [74].

\section{Expression analyses}

In order to begin to assess the possible involvement of these novel genes and families in neural development we analysed the expression of a subset of them by in situ hybridisation in the mouse or fly developing nervous system. Elfn 1 and Elfn 2 show rather complementary expression patterns in the embryonic and postnatal mouse brain (Figure 8). Elfn1 is strongly expressed in interneurons in the hippocampus and cortex while Elfn2 is expressed more broadly in the cortex in presumed glutamatergic neurons and in the hippocampus in pyramidal and granule cells. In the basal ganglia, Elfn 1 is expressed in the globus pallidus, while Elfn2 is expressed more strongly in the other major division, the striatum. These patterns are maintained in adults, according to the Allen Brain Atlas [83]. Based on abundance of cDNAs in the Unigene database, it appears that expression of Elfn2 is quite restricted 


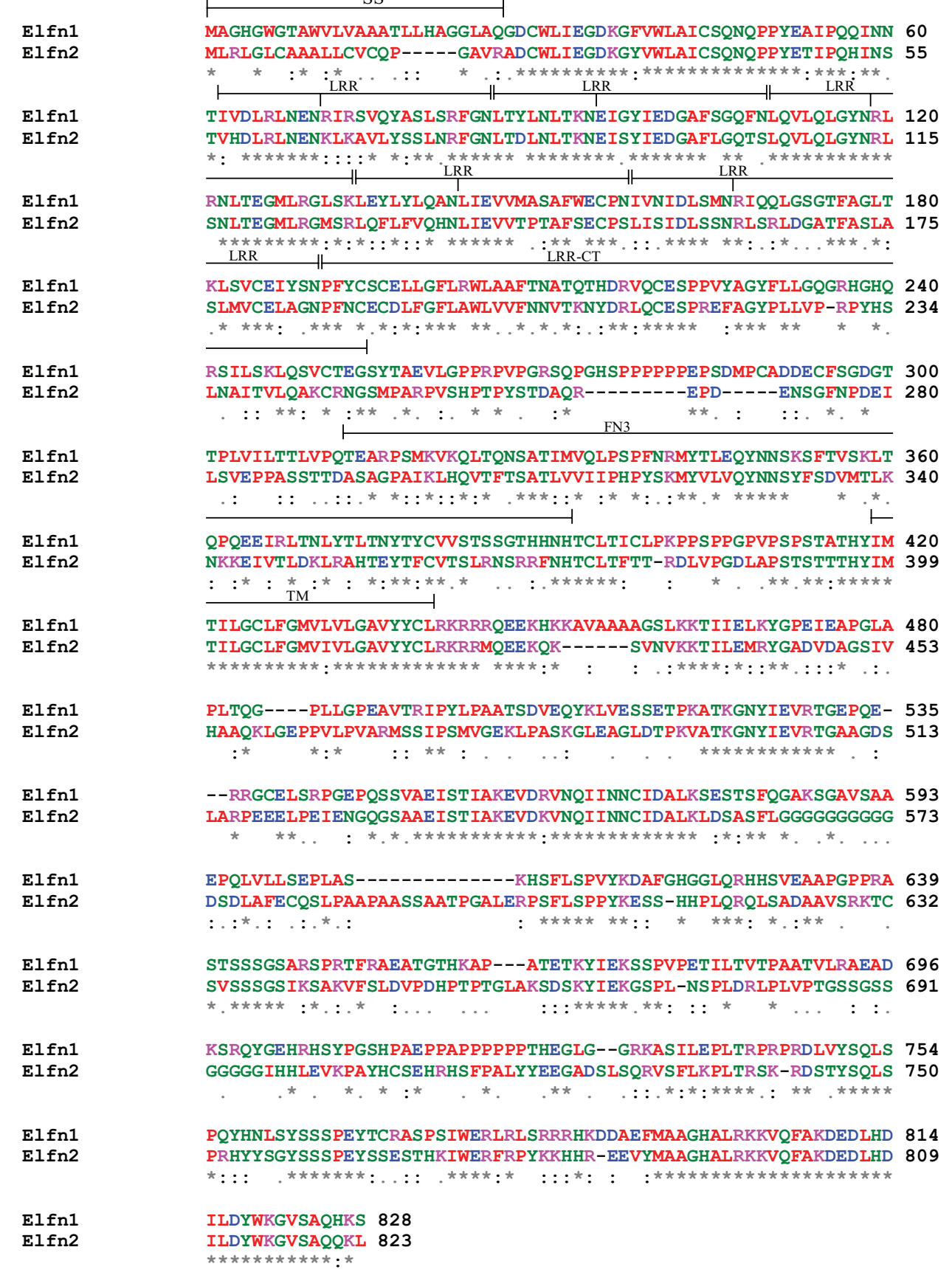

\section{Figure 6}

Alignment of Elfn proteins. Predicted amino acid sequences from Elfn I (A9300 I7N06Rik) and Elfn2 (Lrrc62) from the mouse were aligned with CLUSTALW. Amino acids are colour-coded by chemical properties: blue: acidic; green: hydroxyl/ amine/basic/Q; magenta: basic; red: small, hydrophobic (including aliphatic $Y$ ). Brackets indicate the extent of predicted motifs, including signal sequence (SS), six LRRs (the notch under the bracket indicates the end of the conserved $\mathrm{N}$-terminal portion of each LRR), LRR-CT domain, fibronectin type-3 (FN3) domain and a transmembrane domain (TM). No recognizable LRR-NT domain was predicted. Note that the final LRR comprises the highly conserved N-terminal half-repeat only (consensus: LxxLxxLxLxxN). Identical residues are indicated by an asterisk, highly conservative substitutions by two dots and conservative substitutions by a single dot. 
Lrtm1 Lrtm2 Lrrc38 Lrrc52 Lrrc55 $\mathrm{BC} 004853$

Lrtm1 Lrtm2 $\operatorname{Lrrc3} 8$ Lrrc52 Lrrc55 $\mathrm{BC} 004853$

Lrtm1 Lrtm2 Lrrc38 Lrrc52 Lrrc55 BC004853

Lrtm1 Lrtm2 Lrrc38 Lrrc52 Lrrc55 BC 004853

Lrtm1 Lrtm2 Lrrc38 Lrrc52 Lrrc55 BC004853

Lrtm1 Lrtm2 Lrrc38 Lrrc52 Lrrc55 BC004853

Lrtm1 Lrtm2 Lrrc38 Lrrc52 Lrrc55 BC 004853
SS

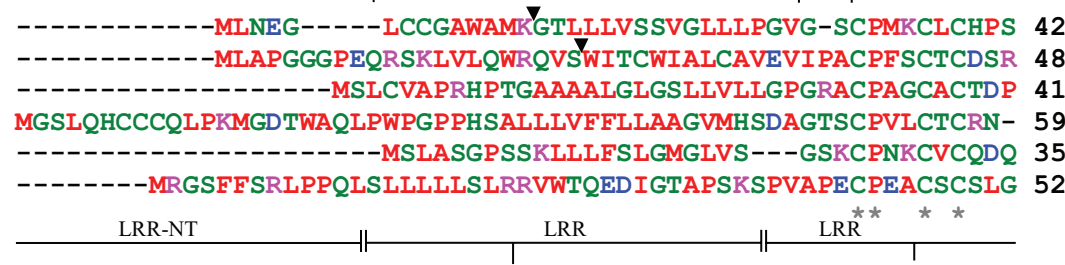
SNSVDCSGQGLSKVPRDLP PWTVTLLLQDNRIHWLPALAFQSVSLLSTLNLSNNSLSNLA 102 SLEVDCSGLGLTTVPPDVPAATOSLLLLNNKLSALPSWAFANLSNLORLDLSNNFLDOLP 108 -HTVDCRDRGLPSVPDPFPLDVRKLLVAGNRIQQI PEDFFIFHGDLVYLDFRNNSLRSLE 100 -QVVDCSNQRLFSVPPDLPMDTRNLSLAHNRIAAVPPGYLTCYMELRVLDLRNNSLMELP 118 --EVACIDLHLTEYPADIPLNTRRLYLNNNKITSLPALQLGFLSDLVYLDCQNNRIREVM 93 -GKANCSALALPAVPADLSWQVRSLLLDHNRVSALPPGAFANAGALLYLDLRENRLRSVH 111

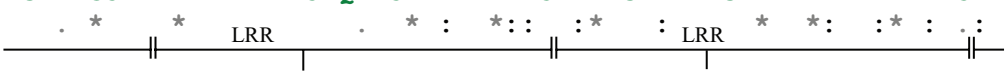

AEAFYGLPHLRVLNVTQNSLLSIESSFAHALPGLRELDLSSN-SLRILPTSLGKPWENLT 161 RS IFEDLVNLTELQLRNNS IRTLDRDLLQHSPLLRHLDLSIN-GLAQLPPGLFDGLLALR 167 EGTFSGSGKLAFLDLSYNNLTOLGAGAFRSAGRLVKLSLANN-HLAGVHEAAFESLESLO 159 PGLFLHAKRLAHLDLSYNNLSHVPADMFREAHGLVHIDLSHNPWLRRVHPQAFQGLVHLR 178 DYTFIGIFRLIYLDLSSNNLTSISPFSFSVLTNLVRLNISHNPHLLYLDKYVFANTTSLR 153 ARAF'WGLGVLQWLDLSSNQLETLPPGTFAPLRALSFLSLAGN-RLALLEPSILGPLPLLR 170

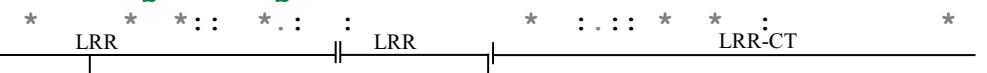

VFAVQQNHLLHLDRELLEAMPKVRLVLLKDNPWICDCHLLGLKLWLERFTFOG-GETDGA 220 SLSLRSNRLQSLDRLTFEPLASLQLLQVGDNPWECDCNLREFKHWLEWFSYRG-GRLDQL 226 VLELNDNNLRSLNVAALDALPALRTVRLDGNPWLCDCDFAHLFSWIQENTSKLPKGLDAI 219 DLDLSYGGLAFLSLEALEGLPGLVTLQIGGNPWVCGCTMEPLLKWLRNRIQRCTADSQLA 238 YLDLRNTGLH I IDHNGFHHLVVLOTLYLSGNPWICNCSFLDFTIHLLVSHMDH-PDAONA 212 VLSLQDNSLSAIEAGLLNNLPALDVLRLHGNPWTCNCALRPLCTWLRKHPRPA-SETETL 229

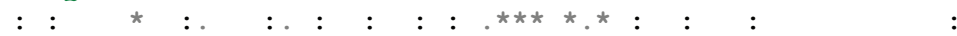

ICRLPEPWOGKALLSI PHELYOPCSLPSODLAPSLVOOPGS----APODAOKSHENSSGO 276 ACTLPKELRGKDMRAVPMEMFNYCSQLEDENNSAGLDAPGPPCTKASPEPPKPKPGAEPE 286

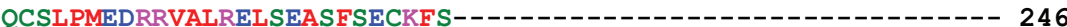
ECRGP PEVEGAPLFSLTEESFKACHLT---------------------------- 265

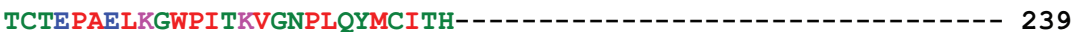

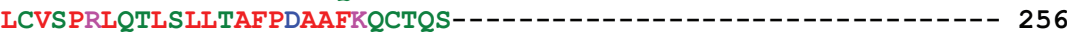
* * : .

QDP-LECEAKPKPKPTNLRHAVATVVITGVVCGIVCLMMLAAAIYGCTYAAITAQYQ--- 332 PEPSTACPOKORYRPVSVRRAIGTVIIAGVVCGIVCIMMVVAAAYGCIYASLMAKYHREL 346 ----------------LSLTDLFIIIFSGVAVSIAAI ISSFFLATVVQCFQRCAP-- 285

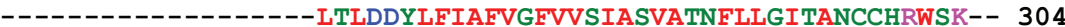
---------------LDQQDYIFLLLIGFCIFAAGTVAAWLTGVCAVLYQNALR-- 278 --------LAARDLAVVYALGPVSFLASLAICLALGSVLTACGARRRRRRRTTVRHLL 306

$$
\text { : : : }
$$

--GRPLASARKSEKMGSKELMDSSSA------------- 356

KKRQPLMGDPEGEHEDQKQISSVA-------------- 370

-------NKDTEDEDDDEDD--------------- 298

-------ANEEEEI----------------------- 311

----TSSGDDTEDETGSRFANQIFRSNTHLGPIRRFPELI 314

RRQLDPEGPPSLEDAGS PVTAAIQA------------- 331

$$
\text { : . }
$$

\section{Figure 7}

Alignment of proteins in Elron cluster. Predicted amino acid sequences from LrtmI, Lrtm2, Lrrc38, Lrrc55, Lrrc52 and BC004853 from the mouse were aligned with CLUSTALW. Brackets indicate the extent of predicted motifs (consensus limits are shown); the notch under the bracket indicates the end of the conserved $\mathrm{N}$-terminal portion of each LRR. Arrowheads denote exon-intron boundaries. The short cytoplasmic domain is poorly conserved, but does contain similarly positioned acidic residues (E/D) in all members. Lrtm I and 2 end in consensus PDZ-binding domains (SSSA/SSVA), underlined. Abbreviations, amino acid colour-code and conservation symbols as in Figure 7. 
to the nervous system (Unigene reference: Mm.323188), while Elfn 1 is also expressed in endocrine and reproductive tissues (Unigene reference: Mm.237102).

Among the genes in the Elron cluster, three (Lrtm1, Lrtm2 and Lrrc55) are expressed in discrete regions of the developing mouse brain, in particular marking different nuclei in the developing thalamus as well as a number of other areas (Figure 9). According to the Allen Brain Atlas, the expression of Lrtm1 declines after development and is practically undetectable in adults. In contrast, Lrtm2 is maintained at high levels in adults in a number of discrete regions including the granule cell layer in the olfactory bulb, the basal ganglia, dorsal thalamus, dentate gyrus, layers $2 / 3$ and 5 in the cortex and Purkinje cells in the cerebellum. Lrrc55 is also maintained at high levels in mitral cells in the olfactory bulb, in the habenula and in layers 4 and $6 a$ in the cortex. Lrrc38 is expressed at lower levels during development (data not shown) but is expressed in a specific pattern in the adult brain, including the CA3 region of the hippocampus and the zona incerta [83]. BC004853 and Lrrc52 do not appear to be expressed in the embryonic or postnatal brain. This result is confirmed by the absence of expression in the Allen Brain Atlas and by analysis of cDNA abundance in the Unigene database, which show that Lrrc52 (Mm.159799) is specific to muscle and testis and that BC004853 (Mm.275228) is almost exclusively expressed by the vesicular organ in the male reproductive system. Similar cDNA abundance data for the other four genes show that LRTM2 (in this case human, Hs.585579) is almost brain-specific, while Lrtm1(Mm.95780), Lrrc38 (Mm.94020) and Lrrc55 (Mm.291095) are also expressed in a small number of other tissues.

The expression of the Elfn genes and of several genes in the Elron cluster is thus consistent with a possible role in specifying neuronal connectivity, especially thalamic and cortical connectivity.

The expression patterns of many of the Drosophila eLRR genes identified in the bioinformatic screen were also examined in the embryo by in situ hybridisation. A summary of the expression patterns we identified and those previously described is presented [see Additional File 7]. We describe here the expression patterns of those novel eLRR genes identified in our survey that include expression in the nervous system (Figure 10). CG7702 is expressed dynamically in the peripheral nervous system (PNS), appearing at stage 11 and disappearing during stage 15. CG40500 is exclusively expressed in the CNS and is restricted to a subset of cells at the ventral midline, beginning during stage 14 and remaining into stage 17 . CG11910 expression is restricted to the most dorsal layer of the CNS in a position consistent with the longitudinal glia. This expression begins at stage 12 and continues throughout embryonic development. CG5888 is expressed from stage 5 throughout the embryo with exception of the anterior tip (data not shown). At stage 15 expression of CG5888 is initiated in a subset of cells in the CNS. CG11136 is expressed in an anteroposterior stripe within the neurogenic region and in the prospective brain lobes during stages 8-10 (data not shown) and in discrete cells at the midline of the CNS during stages 11 and 12 . From stage 11 onwards CG11136 expression is seen predominantly in the somatic musculature.

\section{Discussion}

This study aimed to catalogue the full repertoire of eLRR proteins in the proteomes of worms, flies, mice and humans, to examine their evolutionary relationships and to identify novel proteins and subfamilies that may have important roles in nervous system development.

\section{Methodological issues}

Generating this dataset required identifying all LRR proteins, distinguishing eLRR proteins among this set (i.e., correctly predicting cellular localisation) and analyzing evolutionary relationships across a large set of highly divergent, multi-domain, repetitive proteins in four distantly related species. For all of these tasks we found the use of single programs only partly reliable. It was especially difficult to derive a single set of parameters for any program that would reliably predict the presence of a particular motif or correctly identify orthologues and paralogues for all proteins in the dataset. To overcome this problem we developed an approach of parallel annotation with many different programs, followed by manual curation to arrive at a consensus architecture for each gene, along with a hierarchical clustering method designed to reveal relationships at multiple levels. This is in contrast to the automated one-size-fits-all approaches currently used by some of the large genome databases.

\section{A comprehensive, curated dataset}

Our bioinformatics searches and exhaustive manual curation have yielded what we are confident should be an extremely comprehensive set of eLRR proteins across the four species examined. Rather than employing a series of strict filters we used the combined evidence from a variety of prediction programs and from clustering to distinguish eLRR proteins from intracellular LRR proteins. We think it is therefore unlikely that we have missed many true eLRR genes in any of the organisms. This obviously depends however on the quality and comprehensiveness of the gene predictions in our starting datasets. There may in the first instance be cases of genes that have simply not been predicted at all yet. We also came across numerous cases of mispredicted genes where only a fragment was predicted or where a single ORF was split into two predicted 
Elfn1

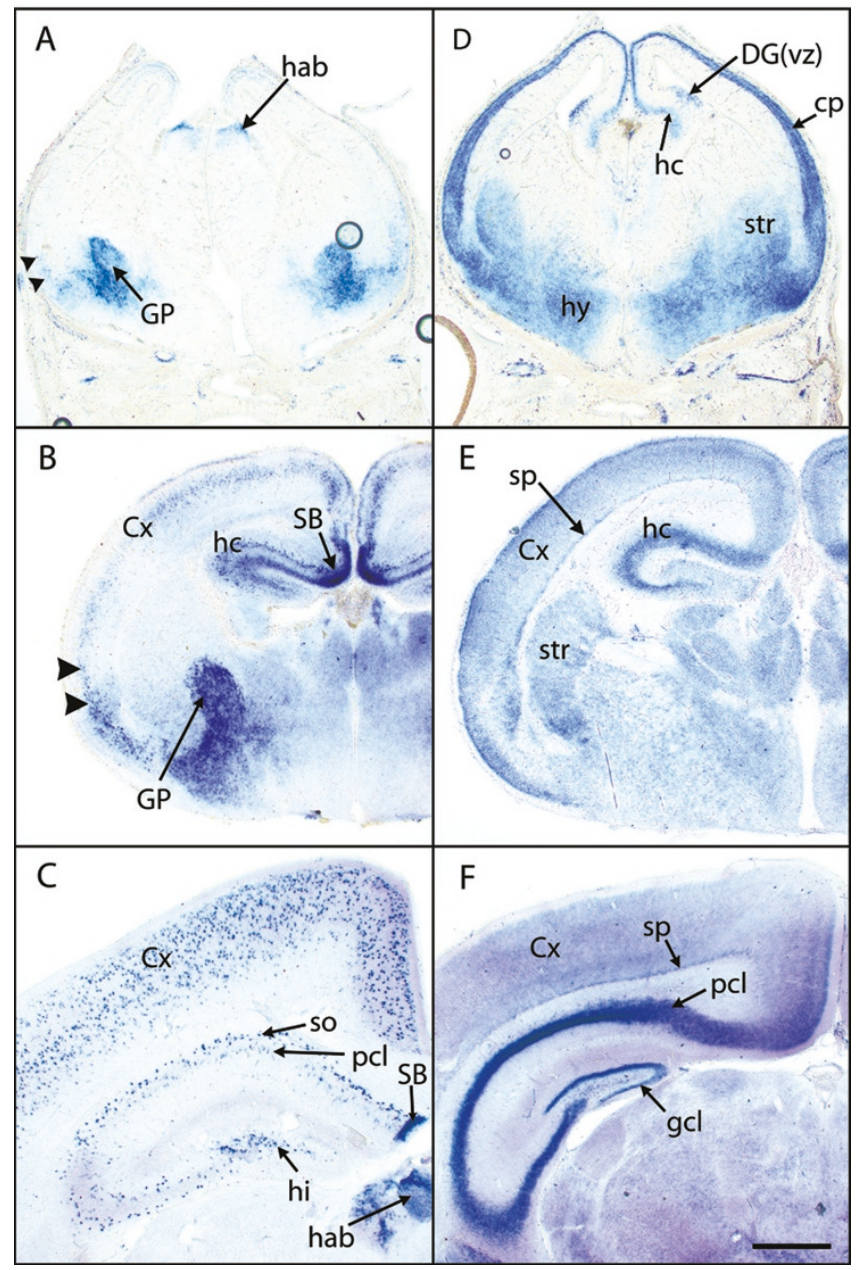

Figure 8

Expression of Elfn genes in developing mouse brain. Expression as defined by RNA in situ hybridisation is shown for ElfnI (A-C) and Elfn2 (D-F) in coronal sections of mouse brain at three ages (embryonic day I5 (EI5), A, D; postnatal day zero (P0), B, E; and postnatal day 9 (P9), C, F). Elfn I is strongly expressed in globus pallidus and interneurons in cortex and hippocampus, while Elfn2 is expressed in striatum and in projection neurons in cortex and hippocampus. Arrowheads in $A$ and $B$ indicate presumed interneurons migrating towards cortex. Abbreviations: cp, cortical plate; Cx, cortex; DG(vz), ventricular zone of dentate gyrus; gcl, granule cell layer (of dentate gyrus); GP, globus pallidus; hab, habenula; hc, hippocampus; hi, hilus (of dentate gyrus); hy, hypothalamus; pcl; pyramidal cell layer (of hippocampus); SB, subiculum; sp, subplate; so, stratum oriens (of hippocampus), str, striatum. Scale bar: EI 5, 200 microns; PO and P9, 500 microns.

genes, for example. In most of these cases the fragments still clustered with other eLRR genes and a full-length sequence was often identifiable from one of the starting datasets. It is difficult to estimate how common such annotation errors are but it is reasonable to expect that they may have caused us to miss a small number of additional eLRR genes or to misclassify some as cytoplasmic.

The manual curation of these sequences has added substantially to the value of this dataset. An appreciable percentage of predicted protein sequences had to be amended in some way to yield what we consider to be the "correct" predicted full-length protein. These corrections were based on various factors including comparison of architectures across orthologues or paralogues, the absence of an expected signal peptide or the location of the predicted start codon with respect to the signal peptide.

Because degenerate or atypical LRRs have been described $[4,49,69]$ that do not match the consensus motifs defined by SMART and Pfam we designed a customised program, LRRscan, to search for a minimal consensus that defines animal extracellular LRRs. We also searched for minimal consensus motifs that define LRR-NT and several varieties of LRR-CT domains found in different types of proteins, including small proteoglycans and G-protein-coupled receptors [4]. These predictions were compared with the results of SMART and Pfam [see Additional File 2] and a consensus architecture was predicted by manual inspection, based on converging evidence. The results match those of proteins with known structures [5-7,70-73,84], significantly better than a combination of SMART and Pfam alone (allowing for semantic differences in whether the final half repeat is counted as one and whether putative LRRs overlapping with NT or CT domains are counted). The predicted transmembrane topologies are also based on converging evidence from multiple programs and have also been subject to expert evaluation. Nevertheless, the architectures presented should be viewed as predictions that will require experimental verification. In particular, the absence of a predicted LRR-NT or LRR-CT domain does not mean there is no domain present that is performing a capping function; there may be additional varieties of such domains that have not yet been defined. In addition, we have chosen a representative isoform for each gene; the database thus contains no information on alternative splice forms or other isoforms that may have differing architectures.

\section{Hierarchical clustering}

The hierarchical clustering method we used gets around the problem of defining a unique set of parameters that is suitable to all proteins and levels of inter-relationship. In most cases, it generates a tree-like structure that reveals relationships across many different levels at once. This is a difficult problem for multiple alignment programs such 


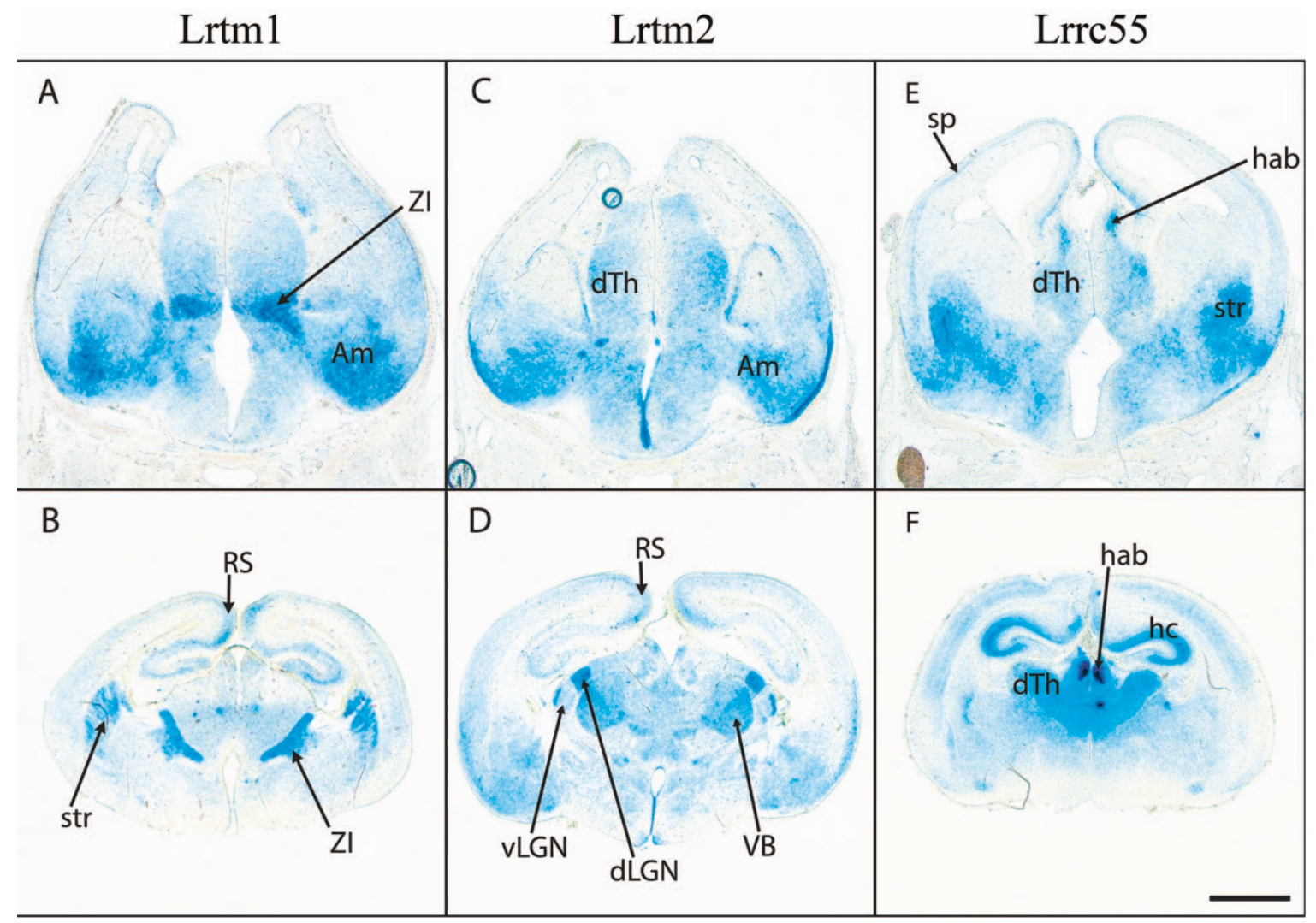

Figure 9

Expression of Elron cluster genes in developing mouse brain. Expression as defined by RNA in situ hybridisation is shown for Lrtm I (A, B), Lrtm2 (C, D) and Lrrc55 (E, F) in coronal sections of mouse brain at two ages (EI5, A, C, E and P0, B, $D, F)$. Differential staining in subsets of thalamic nuclei and across cortex is observed. Abbreviations: Am, amygdala; dLGN, dorsal lateral geniculate nucleus; dTh, dorsal thalamus; hab, habenula; hc, hippocampus; RS, retrosplenial cortex; sp, subplate; str, striatum; vLGN, ventral lateral geniculate nucleus; ZI, zona incerta. Scale bar: EI 5, 200 microns; P0, 500 microns.

as CLUSTALW or T-COFFEE, which work well for closely related proteins but which are not designed to compare highly divergent proteins with differing architectures. Previous attempts using multiple alignment programs to derive a phylogenetic tree across many eLRR subfamilies at once contain numerous differences from our results and from known relationships $[3,85]$.

In some cases, the results of TribeMCL depart from the expected hierarchical relationship. This is the case for the extended LRR_Tollkin group of proteins, including many proteins characterized by an eLRR domain but lacking an obvious TIR domain. While the clustering of these proteins with the TLR group is quite convincing, based on direct inspection of the BLAST results, it is extremely difficult, indeed impossible with these data, to discern more discrete relationships within this large family. The reasons for the anomalous hierarchical clustering results with these genes may relate to the large number of LRRs present in these proteins and the very slight differences in pairwise similarities across the group. At different levels of stringency small differences in BLAST scores may be amplified by the TribeMCL algorithm to result in membership of different clusters that do not share the expected hierarchical relationship. Attempts to resolve the phylogeny of all the genes in this group using the T-COFFEE multiple alignment program were no more enlightening, resulting in a starburst pattern where the roots of each subfamily are too close to each other to resolve (data not shown). Despite these limitations, the TribeMCL analysis has revealed a group of eLRR proteins that are clearly more related to the TLR proteins than to other LRR_Only proteins.

\section{Nomenclature}

The current nomenclature of eLRR proteins is very confusing, with multiple synonyms for many genes [see Additional File 3], many of which do not give accurate information on relationships. For example, there is a large number of proteins designated LrrcX, where $\mathrm{X}$ is a 


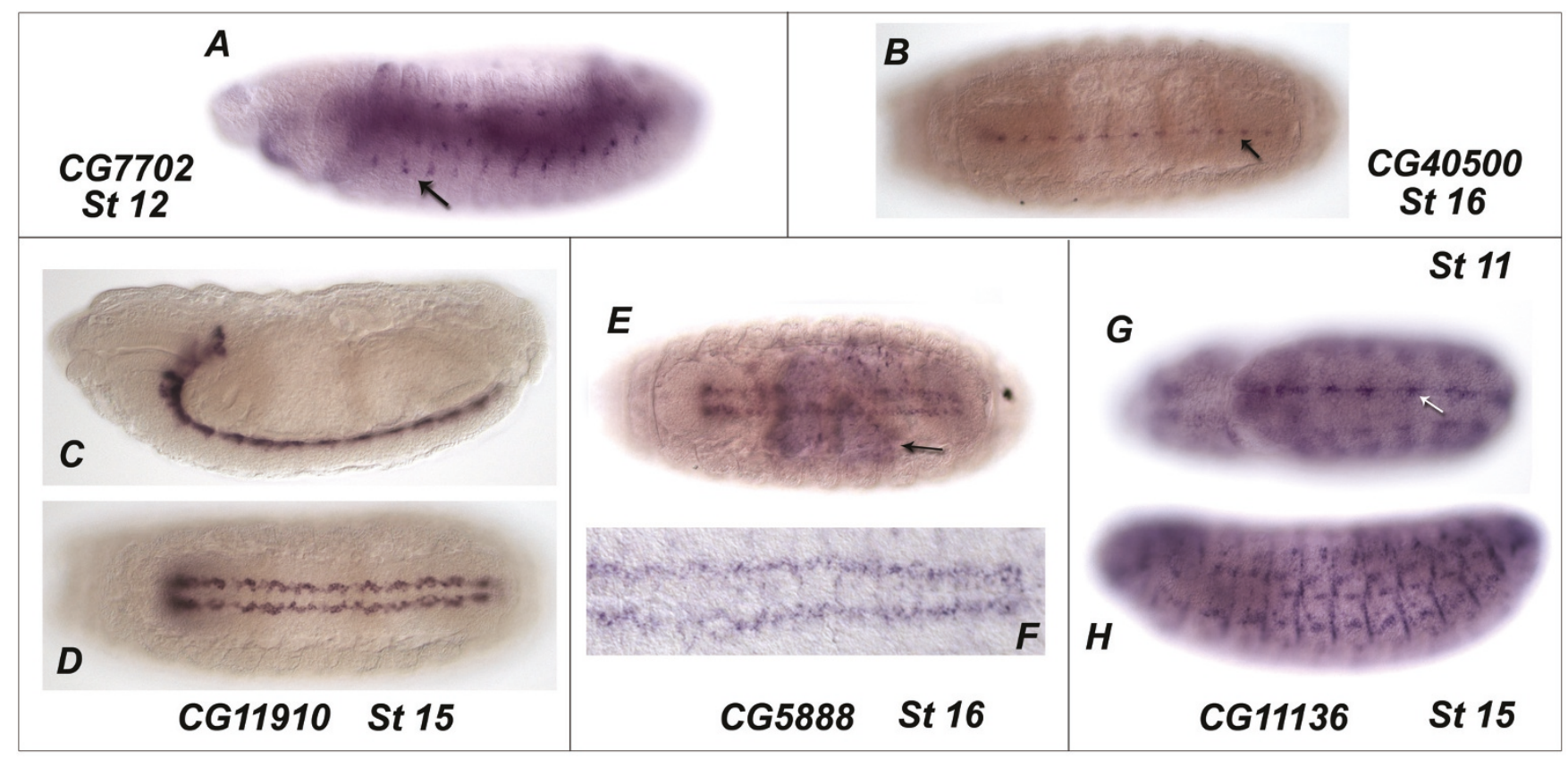

Figure 10

Expression of novel eLRR genes in the Drosophila embryo. (A) A lateral view of a stage 12 embryo showing expression of CG7702 in the midgut and the peripheral nervous system, PNS expression is indicated by a black arrow. (B) CG40500 expression in a stage 16 embryo, expression can be seen at the midline (indicated by a black arrow). (C and D) Lateral and ventral views, respectively, of a stage 15 embryo showing CGI 1910 expression in the central nervous system. (E) A stage 16 embryo with CG5888 expression in the CNS and midgut chamber, midgut chamber is indicated by a black arrow. (F) A dissected ventral nerve cord fillet with CG5888 expression (shown at 400x magnification). (G) A stage II embryo showing CG I I / 36 expression at the midline, indicated by a white arrow and $(\mathrm{H})$ a stage I 5 embryo showing expression of CG I I /36 in the somatic musculature. All whole embryos are shown at $200 \times$ magnification. In all views anterior is to the left, in all lateral views dorsal is at the top, $B, D$ and $E$ show ventral views and $G$ shows a dorsal view.

number. These names were apparently derived from largescale genome projects and do not represent a specific subfamily of related proteins. For that reason we have proposed the names Elfn 1 and 2 for one novel subfamily. We also identify another discrete cluster of six "novel" proteins (which we refer to as the Elron cluster), although whether they represent a true subfamily is open to debate. In addition, some novel proteins that group into small subfamilies with Lrrc21/Pal, Chad, Lrrc3 and Tpbg/5T4 [see Additional File 3, Figures 3 and 4] could be given names to reflect that fact. Finally, while Lrrn1, 2 and 3 (also known as NLRR1, 5 and 3) form a subfamily, the recently named NLRR4 [46] is not in fact a member of this subfamily. It does not have the Ig domain present in these genes and does not cluster with those genes at any parameters.

\section{Comparative analyses of major groups}

For the purposes of some of the analyses we split the eLRR proteins into four groups, based on architecture and clustering results. The LRR_Ig/FN3 group includes the largest percentage of mammal-specific subfamilies, many with multiple members. Almost all of the proteins in this group are associated with the membrane, either type I TM or GPI-linked. The majority of these subfamilies (including Ntrks, Lrfns/Salms, Flrts, Lrigs, Netrin-G ligands, and Lingo proteins) show discrete expression in the nervous system and many of them have been shown to have functions in neural development [3] and/or have been implicated in neurological or psychiatric disease [49]. Expansion of this class of proteins is thus correlated with the evolution of the complex mammalian brain and plausibly contributed to it by providing the requisite specificity of cellular interactions to mediate a large number of selective connectivity decisions. We have identified a novel mammal-specific LRR_FN3 subfamily, the Elfn proteins, with discrete nervous system expression.

The LRR_Only group shows independent diversification in flies and mammals, with a large number of singletons (unclustered proteins), suggesting rapid sequence divergence. This group also contains a number of proteins implicated in nervous system development or function including the Nogo-receptor, Lrrtm and Slitrk families, 
and connectin, Gp150, Tpbg/5T4 and Nyx for example. We have identified six proteins in another novel mammal-specific cluster, the Elron cluster, several of which show highly suggestive expression patterns in the developing nervous system. We have also discovered a number of novel fly proteins in this class that are similarly discretely expressed in the embryonic nervous system.

The LRR_Tollkin group shows a different pattern of evolution, with parallel expansions in flies and mammals, of both the Toll-like receptor genes and of the genes that cluster with them. In mammals, the latter include Cpn2, Gp5 and Lrrc15 (Lib), which form a subcluster and which are also arrayed in tandem on chromosome 3 in humans (16 in mouse). These proteins have diverse binding partners and biochemical functions but are all involved in inflammation in some way: as a regulatory subunit of carboxypeptidase [86], as a component of the platelet glycoprotein complex (which also contains the eLRR proteins GP1b $\alpha$ and GP1b $\beta[87])$, and as a mediator of the glial response to $\beta$-amyloid [88], respectively. They form a slightly larger cluster with Igfals, the acid-labile subunit of insulin growth factor, which regulates IGF signaling [89] and with the novel gene KIAA0644, which has an FN3 domain in addition to the LRR domain. Lrrc32/GARP [90] and its paralogue Lrrc33 also cluster with this group at $\mathrm{e}^{-}$ 40 , level 1, but not at some lower levels. The novel fly gene CG7509 also clusters with this group at $\mathrm{e}^{-40}$, level 1 and with other fly genes including chaoptin at some other levels. Whether it can be said to be directly orthologous to any (or all) of these mammalian proteins is hard to determine. The other fly genes in this group are mostly novel and include CG40500-PD, which has an FN3 domain and which shows very discrete expression in the midline of the embryonic nervous system.

The LRR_Other group is an arbitrary default group as it contains many unrelated genes or subfamilies. Nevertheless, it is interesting to note that this group contains the highest percentage of genes with orthologues across all species, including worms (e.g., the slits and peroxidasins and some of the seven-transmembrane hormone receptors). This group also includes the mammalian Lgi subfamily, recently implicated in epilepsy and myelination.

\section{Human-mouse differences}

Only a small number of proteins are specific to either human or mouse. There are two cases where there are genes in humans that are not represented in mouse that both seem to be caused by specific loss in mice, rather than representing human-specific genes. The human gene synleurin appears to have been pseudogenised in rodents, although it is present in many other species besides humans (including dog, cow and chick, for example). Also, MXRA5 (or adlican), a paralogue of the large secreted protein Igsf10, is not detectable in the mouse genome (but is present in cow, dog and opossum, for example).

There is also a small number of examples where there has been independent expansion of subfamilies in either humans or mice. These include the Toll-like receptors TLR10 in humans and TLR11, 12 and 13 in the mouse. They also include the unusual subfamily of LRRC37 genes, which is represented by a single gene in the mouse (called Lrrc37a) but multiple, highly related genes in humans (LRRC37A, A2 and A3 and LRRC37B, as well as a number of other partial duplicates lacking LRRs). These are located in tandem on chromosome 17 and have arisen from multiple duplications of the BRCA1 region in primates [91]. The extracellular domains of these TM proteins are characterised by six predicted LRRs but these make up only a small fraction of the overall protein, which is highly variable in length. The functions and expression patterns of these unusual proteins are unknown.

\section{Conclusion}

This survey presents a comprehensive overview of the repertoire of eLRR proteins in various species and their interrelationships. As such, it provides the necessary foundation for a systematic analysis of the functions of this class of genes, which are likely to include prominently neural development, innate immunity and inflammation. In particular, expansion of the eLRR proteome is correlated with increasing complexity of the nervous system. Given the functions and discrete expression patterns of many known members, it seems likely that this superfamily, including the novel proteins identified here, could provide the requisite specificity of cellular interactions to mediate a large number of selective connectivity decisions.

\section{Methods}

\section{Database pipeline}

Protein sequences for all four species were retrieved from the Ensembl FTP site: Mouse release 36 NCBI m34 assembly (36471 sequences); Human release 36, NCBI 35 assembly (33869 sequences); Worm release 37, Wormbase 150 dataset (26032 sequences); Fly release 37, BDGP assembly release 4 (19369 sequences). In addition, 68627 mouse and 57366 human protein sequences were downloaded from the International Protein Index, version 3.14. A further 24273 human and 19258 mouse protein sequences were retrieved through the web interface [92] from the August 2006 version of the Mammalian Gene Collection. We also included a further 879 sequences comprising many from an older version of the Mammalian Gene Collection (February 2006) that were absent from the August 2006 release as well as several more 
added manually. All sequences were stored for easy access in a MySQL database.

The data set was reduced through use of a small Perl script that filters out duplicate copies of sequences for each species and keeps either the Ensembl version or an entry with a flag indicating its preference after manual curation. The non-redundant data sets for mouse, human, worm, and fly contained 85991, 74866, 22698, and 16857 sequences, respectively.

These sequences were subjected to an all-against-all Blast search (NCBI BlastP, version 2.2.12) carried out on a high-performance Linux cluster. An expectation cut-off of 0.1 was specified, and the top 200 hits for each search in tab-delimited format (-m8) were reported. The Blast results were parsed with the mcxdeblast tool using expectation cut-offs from $e^{-10}$ to $e^{-40}$ and formatted for clustering with the mcxassemble tool (options -q -r max -map -b), both part of the MCL package (version 1.005, 05-272). Each output was then subjected to Markov clustering with the MCL program using inflation parameters ranging from 1.2 to 5 . The program Tribe-families was then run to produce the final clusters.

For proteins from the IPI and MGC set that did not have gene IDs assigned, we produced alignments using T-Coffee (version 3.93) with their best Blast hits. If sequences with matching protein names were found that are fully contained in another one or showed identity over at least $95 \%$ and sequence difference of maximal $15 \%$ we transferred Ensembl gene ID annotation where available. Through this, 2490 sequences from mouse and 1458 sequences from human were assigned Ensembl gene IDs. The gene information was used to remove isoforms from the clusters: only the protein with the longest sequence was kept for each gene. In some cases, where dubious excessive amino acids seem to have been added to a sequence, manual curation was necessary to overwrite this behavior and select proteins that seemed biologically more plausible.

For prediction of architecture we used HMMpfam of the HMMER package (version 2.3.2) [93], together with the SMART (release 25 Nov. 2004) and Pfam (version 19.0) HMM libraries. Transmembrane predictions were produced by the programs TMHMM (version 2.0 [58]), HMMTOP (version 2.1 [63]), and TMPred [64]. Signal Sequence analysis was carried out using SignalP (version 3.0 [59]) and GPI-link results calculated by the BIG-PI program [65] were obtained for human [94] and fly [95]. In addition, information about the genomic location and synonyms for a gene were retrieved from Ensembl, MGI, Wormbase and Flybase. Clustering and annotation infor- mation were combined into a large spreadsheet for the final output.

\section{LRRscan}

Based on a number of published studies $[1,4,49,96]$ and our own inspection of the sequences in our dataset we defined the minimal $\mathrm{N}$-terminal part of a single extracellular-type LRR as: LxxLxLxxN. This is followed by a C-terminal part of each LRR of typically 10-21 amino acids that are quite variable. Consensus sequences for the LRR capping domains (LRR-NT at the N-terminus and LRR-CT at the C-terminus) have been defined by [4], including three different consensus sequences for the LRR-CT domain, derived from different classes of proteins. LRRCT1 is the most common type, LRR-CT2 is found in small proteoglycans and LRR-CT3 in G-protein-coupled receptors:

LRR-CT1 domain: P(w/f)xCxCxoxWLxxw(9-24)oxC(918)CxxP

LRR-CT2 domain: nI(s/t)xogxxdFCxoxxxxo(4-5)y(4)Lxx Npo(6)PxxfxCo

LRR-CT3 domain: LxxAxL(s/t)YPSHCCAFxN(6-19)nosx CnxsxxR...

LRR-NT domain: (7-10)CP(2-5)CxC(4-17)oxC(2-4)ox xoPxxoP

" $\mathrm{x}$ " represents any residue and "o" a non-polar residue [4].

We derived a minimal consensus sequence from each of the above and designed a new program, LRRscan, to search for these sequences as well as the minimal LRR defined above. The search for LRR-NTs and LRR-CTs focuses exclusively on the cysteines, which are the most conserved amino acids in these motifs. The regular expressions applied are as follows:

LRR: L..L.L..N. $\{10,21\}$

LRR-NT: C. $\{2,8\}$ C.C. $\{6,19\}$ C. $\{11,15\}$

LRR-CT1: ...C.C. $\{19,34\}$ C. $\{9,18\}$ C. $\{3\}$

LRR-CT1_short: ...C.C. $\{19,34\}$ C. $\{22\}$

LRR-CT2: . $\{10\}$ C. $\{30,31\}$ C.

LRR-CT3: ..CC. $\{14,27\}$ C. $\{6\}$

(Each dot represents any single letter, numbers in curly brackets indicate a repeat frequency, either exact or as a range where two numbers are given. Dots at the beginning 
or end of a domain denote spacing from the start or end of other motifs, including LRRs).

LRRscan was written in Perl and has been especially designed for the detection of LRR motifs. Input consists of a sequence file in FASTA format as well as search parameters. Each sequence is scanned for patterns, specified as strings, and alternative amino acids for certain positions, specified as triplets comprising position, alternative amino acid and score. The LRR pattern used in our search was 'LxxLxLxxN', where a small 'x' acts as a placeholder for any amino acid. The alternative options were amino acids A, I, V, F, G, M, or W for any of the leucines and C, S, or T instead of the asparagine. Each exact match between an amino acid and the search pattern produces a score of 1 , whereas a match to an alternative letter only scores 0.4 . The scores are summed up over the length of the pattern and a minimum score of 2 would lead to further consideration of the sequence region. To allow for maximum sensitivity an exhaustive search is carried out, i.e., all patterns that match the search criteria are initially captured even if they overlap.

In the next step the regions are grouped into stretches of LRRs located within a specific distance from each other, in our case allowing for a gap of 20 to 30 amino acids between starts of pattern. Within a sequence of LRRs the overlaps are removed by only keeping the highest scoring regions. However, overlaps between sets of LRRs are allowed in the LRRscan output. Such occurrences are indicated in the output by a backward shift in the sequence location, i.e. the end of one stretch of LRRs might be printed again at the beginning of the next one. This is usually interpreted in the manual curation process as an insertion in the LRR domain. Each sequence is also scanned for a minimal LRR-NT pattern in the upstream sequence and for a minimal LRR-CT pattern (one of three possible types) in the downstream sequence. These are allowed to overlap with predicted LRRs to maximise the detection rate. The presence of additional elements from the more complete consensus sequences defined by Kajava was considered as supportive evidence in the manual curation process. Two alternative types of LRR-NT have been proposed, with different numbers of cysteines [97]. We found it difficult to ascertain whether these were really evolutionarily distinct or whether some cysteines were simply not well conserved and SMART and Pfam can detect both types. For these reasons we have not attempted to distinguish between these putative types of LRR-NT. Similarly, some LRR-CT domains could not be categorized definitively as CT1 or CT2 subtypes; these are denoted as LRR-CT in Table 1 and [see Additional File 3].

The output from LRRscan reports the sequence that was searched and the positions of the motifs found followed by the sequence of the motifs themselves. The LRRs are numbered sequentially (within a set of grouped LRRs) and spaced to easily distinguish the well-conserved $\mathrm{N}$-terminal from the more variable C-terminal part [see Additional File 2]. We ran LRRscan on a set of protein sequences for which HMMpfam had predicted LRR motifs already (using the PFAM and SMART databases). The high sensitivity might result in an excessive number of false positives if applied to other sequences, but our goal was to further increase the detection rate of LRRs in sequences that showed an initial sign of LRR occurrence. A summary graphical output was generated for the output of each program for each sequence and aligned for easy comparison [see Additional File 2]. Through extensive manual curation a consensus predicted architecture was produced. It was found during manual curation that the cysteine residues in the NT and CT domains were not always positioned strictly according to the above consensuses and some flexibility was allowed for in these cases. We counted the final half repeat before the CT domain as one and did not include putative LRRs that overlapped with well-defined LRR-NT or LRR-CT domains in the total number of repeats.

\section{RNA in situ hybridization}

Please [see Additional File 8] for details.

\section{Abbreviations}

ELRR, extracellular leucine-rich repeat; FN3, fibronectintype 3; GPI, glycosyl phosphatidyl inositol; Ig, immunoglobulin; LRR, leucine-rich repeat; LRR-CT, leucine-rich repeat C-terminal domain; LRR-NT, leucine-rich repeat Nterminal domain; TIR, Toll/IL-1 receptor; Tlr, Toll-like receptor; $\mathrm{TM}$, transmembrane.

\section{Authors' contributions}

The project was designed by KM and GT. SO'K and KH carried out bioinformatics analyses. JD, KW, TO and SM performed analyses on mouse genes. SA performed analyses on fly genes. The manuscript was prepared by KM, JD, $\mathrm{KW}, \mathrm{SA}, \mathrm{KH}$ and GT. All authors read and approved the final manuscript.

\section{Additional material}

\section{Additional file 1}

Curated sequences of eLRR proteins. List of curated sequences of eLRR proteins in FASTA format.

Click here for file

[http://www.biomedcentral.com/content/supplementary/1471-

2164-8-320-S1.doc] 


\section{Additional file 2}

LRRscan_out.html. Graphical comparison of HMMpfam and LRRscan results. A compressed archive (lrr_plots.tar.gz) containing 372 images in Portable Network Graphics (PNG) format, an information file (OOREADME.txt) and two HTML-formatted pages, one with output from LRRscan (LRRscan_out.html) and one that links all the images together (00plots.html). After downloading, the archive must be to uncompressed and unpacked. Most modern operating systems (e.g. Windows XP, Mac OS X) will do this automatically when double-clicking on the file. Alternatively, you can use the free tool 'Stuffit Expander' (http:// www.stuffit.com) or your favourite unpacker. On Linux or Unix systems apply the following command: tar zxf lrr_plots.tar.gz. Please note that some browsers might uncompress the file during download without changing the file ending. If you have trouble unpacking the file try renaming it to lrr_plots.tar and double-click on it again. Unpacking the archive creates a new folder (lrr_plots) in which you can find a file called '00plots.html'. Open this file in a web-browser, either by double-clicking onto it or by using the 'File->Open File' menu (or equivalent) of your browser. This will bring up a web-page with plots of LRR motifs for 372 proteins. If you click on an image you can see the text output from LRRscan in a new window.

Click here for file

[http://www.biomedcentral.com/content/supplementary/14712164-8-320-S2.gz]

\section{Additional file 3}

Table S1. Complete list of genes, clustered at $e^{-40}$.

Click here for file

[http://www.biomedcentral.com/content/supplementary/14712164-8-320-S3.xls]

\section{Additional file 4}

Table S2. Complete list of genes, clustered at $e^{-25}$.

Click here for file

[http://www.biomedcentral.com/content/supplementary/14712164-8-320-S4.xls]

\section{Additional file 5}

Table S3. Complete list of genes, clustered at $e^{-10}$.

Click here for file

[http://www.biomedcentral.com/content/supplementary/14712164-8-320-S5.xls]

\section{Additional file 6}

Table S4. List of clusters used in Figure 5.

Click here for file

[http://www.biomedcentral.com/content/supplementary/14712164-8-320-S6.xls]

\section{Additional file 7}

Table S5. Summary of fly gene expression

Click here for file

[http://www.biomedcentral.com/content/supplementary/14712164-8-320-S7.xls]

\section{Additional file 8}

Additional methods. In situ hybridisation protocols

Click here for file

[http://www.biomedcentral.com/content/supplementary/14712164-8-320-S8.doc]

\section{Acknowledgements}

$\mathrm{SA}, \mathrm{KH}$ and SO'K contributed equally to this work. We thank the Trinity Center For High Performance Computing. The Linux clusters are provided by the IITAC research project, funded by the HEA PRTLI Cycle 3 program, through the National Development Plan and are managed by staff at

TCHPC. We are very grateful to the following people for providing us with programmes or results: TMHMM: Anders Krogh \& Kristoffer Rapacki and SignalP: Soren Brunak, all at the Centre For Biological Sequence Analysis at Technical University Denmark. For TMPred: Kay Hoffman; BIG-PI program prediction results: Birgit Eisenhaber; SMART Hidden Markov Model library: Peer Bork. We thank Stijn van Dongen and Anton Enright for providing MCL and Tribe-families and for helpful discussions and advice. SA, SO'K and TO were supported by a grant from the Wellcome Trust to KJM and GT (075264/A/04/Z). KJM, JD, KW and SM are supported by SFI grant (0I/F.I/ B006). $\mathrm{KH}$ is supported by the SFI AOIP programme.

\section{References}

I. Kobe $B, K a j a v a A V$ : The leucine-rich repeat as a protein recognition motif. Curr Opin Struct Biol 200I, I I:725-32.

2. Nurnberger T, Brunner F, Kemmerling B, Piater L: Innate immunity in plants and animals: striking similarities and obvious differences. Immunol Rev 2004, 198:249-66.

3. Chen Y, Aulia S, Li L, Tang BL: AMIGO and friends: An emerging family of brain-enriched, neuronal growth modulating, type I transmembrane proteins with leucine-rich repeats (LRR) and cell adhesion molecule motifs. Brain Res Brain Res Rev 2006, 5 I:265-74.

4. Kajava AV: Structural diversity of leucine-rich repeat proteins. J Mol Biol 1998, 277:519-27.

5. Bell JK, Botos I, Hall PR, Askins J, Shiloach J, Segal DM, Davies DR: The molecular structure of the Toll-like receptor 3 ligandbinding domain. Proc Natl Acad Sci USA 2005, 102:10976-80.

6. Mosyak L, Wood A, Dwyer B, Buddha M, Johnson M, Aulabaugh A, Zhong X, Presman E, Benard S, Kelleher K, et al.: The structure of the LINGO-I ectodomain, a module implicated in CNS repair inhibition. Journal of Cell Biology 2006, 28 I:36378-90.

7. He XL, Bazan JF, McDermott G, Park JB, Wang K, Tessier-Lavigne M, He Z, Garcia KC: Structure of the Nogo receptor ectodomain: a recognition module implicated in myelin inhibition. Neuron 2003, 38: $177-85$.

8. West AP, Koblansky AA, Ghosh S: Recognition and signaling by toll-like receptors. Annu Rev Cell Dev Biol 2006, 22:409-37.

9. Lazzaro BP, Sackton TB, Clark AG: Genetic Variation inDrosophila melanogaster Resistance to Infection: A Comparison Across Bacteria. Genetics 2006, 174:1539-54.

10. Anderson KV, Bokla L, Nusslein-Volhard C: Establishment of dorsal-ventral polarity in the Drosophila embryo: the induction of polarity by the Toll gene product. Cell 1985, 42:79|-8.

II. Halfon MS, Hashimoto C, Keshishian H: The Drosophila toll gene functions zygotically and is necessary for proper motoneuron and muscle development. Dev Biol 1995, 169:151-67.

12. Gangloff M, Weber AN, Gibbard RJ, Gay NJ: Evolutionary relationships, but functional differences, between the Drosophila and human Toll-like receptor families. Biochem Soc Trans 2003, $31: 659-63$.

13. Kambris Z, Hoffmann JA, Imler JL, Capovilla M: Tissue and stagespecific expression of the Tolls in Drosophila embryos. Gene Expr Patterns 2002, 2:3।I-7.

14. Pujol N, Link EM, Liu LX, Kurz CL, Alloing G, Tan MW, Ray KP, Solari $R$, Johnson $C D$, Ewbank Jj: A reverse genetic analysis of components of the Toll signaling pathway in Caenorhabditis elegans. Curr Biol 200I, II:809-2I.

15. Kaufman A, Dror G, Meilijson I, Ruppin E: Gene Expression of Caenorhabditis elegans Neurons Carries Information on Their Synaptic Connectivity. PLoS Comput Biol 2006, 2:e I67.

16. Wadachi $R$, Hargreaves KM: Trigeminal nociceptors express TLR-4 and CD 14: a mechanism for pain due to infection. $J$ Dent Res 2006, 85:49-53.

17. Ma Y, Li J, Chiu I, Wang Y, Sloane JA, Lu J, Kosaras B, Sidman RL, Volpe IJ, Vartanian T: Toll-like receptor 8 functions as a negative regulator of neurite outgrowth and inducer of neuronal apoptosis. Journal of Cell Biology 2006, 175:209-2I5. 
18. Van Vactor D Jr, Krantz DE, Reinke R, Zipursky SL: Analysis of mutants in chaoptin, a photoreceptor cell-specific glycoprotein in Drosophila, reveals its role in cellular morphogenesis. Cell 1988, 52:28I-90.

19. Nose A, Takeichi M, Goodman CS: Ectopic expression of connectin reveals a repulsive function during growth cone guidance and synapse formation. Neuron 1994, I 3:525-39.

20. Kidd T, Bland KS, Goodman CS: Slit is the midline repellent for the robo receptor in Drosophila. Cell 1999, 96:785-94.

21. Tsuji L, Yamashita T, Kubo T, Madura T, Tanaka H, Hosokawa K, Tohyama M: FLRT3, a cell surface molecule containing LRR repeats and a FNIII domain, promotes neurite outgrowth. Biochem Biophys Res Commun 2004, 3 I 3: 1086-91.

22. Aruga J, Mikoshiba K: Identification and characterization of Slitrk, a novel neuronal transmembrane protein family controlling neurite outgrowth. Mol Cell Neurosci 2003, 24: I I 7-29.

23. Lauren J, Airaksinen MS, Saarma M, Timmusk T: A novel gene family encoding leucine-rich repeat transmembrane proteins differentially expressed in the nervous system. Genomics 2003 , 8I:4II-2I.

24. Lin JC, Ho WH, Gurney A, Rosenthal A: The netrin-GI ligand NGL-I promotes the outgrowth of thalamocortical axons. Nat Neurosci 2003, 6:1270-6.

25. Kuja-Panula J, Kiiltomaki M, Yamashiro T, Rouhiainen A, Rauvala H: AMIGO, a transmembrane protein implicated in axon tract development, defines a novel protein family with leucinerich repeats. J Cell Biol 2003, I60:963-73.

26. Ko J, Kim S, Chung HS, Kim K, Han K, Kim H, Jun H, Kaang BK, Kim E: SALM synaptic cell adhesion-like molecules regulate the differentiation of excitatory synapses. Neuron 2006, 50:233-45.

27. Kim S, Burette A, Chung HS, Kwon SK, Woo J, Lee HW, Kim K, Kim $H$, Weinberg RJ, Kim E: NGL family PSD-95-interacting adhesion molecules regulate excitatory synapse formation. Nat Neurosci 2006, 9:1294-301.

28. Karaulanov EE, Bottcher RT, Niehrs C: A role for fibronectin-leucine-rich transmembrane cell-surface proteins in homotypic cell adhesion. EMBO Rep 2006, 7:283-90.

29. Nose A, Umeda T, Takeichi M: Neuromuscular target recognition by a homophilic interaction of connectin cell adhesion molecules in Drosophila. Development 1997, I 24: |433-4I.

30. Shinza-Kameda M, Takasu E, Sakurai K, Hayashi S, Nose A: Regulation of layer-specific targeting by reciprocal expression of a cell adhesion molecule, capricious. Neuron 2006, 49:205-I3.

3I. Park JB, Yiu G, Kaneko S, Wang J, Chang J, He XL, Garcia KC, He Z: A TNF receptor family member, TROY, is a coreceptor with Nogo receptor in mediating the inhibitory activity of myelin inhibitors. Neuron 2005, 45:345-5I.

32. Shao Z, Browning JL, Lee X, Scott ML, Shulga-Morskaya S, Allaire N, Thill G, Levesque M, Sah D, McCoy JM, et al:: TAJ/TROY, an orphan TNF receptor family member, binds Nogo-66 receptor I and regulates axonal regeneration. Neuron 2005, 45:353-9.

33. Wang KC, Kim JA, Sivasankaran R, Segal R, He Z: P75 interacts with the Nogo receptor as a co-receptor for Nogo, MAG and OMgp. Nature 2002, 420:74-8

34. Wang KC, Koprivica V, Kim JA, Sivasankaran R, Guo Y, Neve RL, He $\mathrm{Z}$ : Oligodendrocyte-myelin glycoprotein is a Nogo receptor ligand that inhibits neurite outgrowth. Nature 2002, 4 I 7:94|-4

35. Conover JC, Yancopoulos GD: Neurotrophin regulation of the developing nervous system: analyses of knockout mice. Rev Neurosci 1997, 8: 13-27.

36. Fukata $Y$, Adesnik H, Iwanaga T, Bredt DS, Nicoll RA, Fukata M: Epilepsy-related ligand/receptor complex LGII and ADAM22 regulate synaptic transmission. Science 2006, 3 I 3: I792-5.

37. Ghiglione C, Carraway KL 3rd, Amundadottir LT, Boswell RE, Perrimon N, Duffy JB: The transmembrane molecule kekkon I acts in a feedback loop to negatively regulate the activity of the Drosophila EGF receptor during oogenesis. Cell 1999, 96:847-56.

38. Fukamachi K, Matsuoka Y, Ohno H, Hamaguchi T, Tsuda H: Neuronal leucine-rich repeat protein-3 amplifies MAPK activation by epidermal growth factor through a carboxyl-terminal region containing endocytosis motifs. I Biol Chem 2002, 277:43549-52
39. Bottcher RT, Pollet N, Delius H, Niehrs C: The transmembrane protein XFLRT3 forms a complex with FGF receptors and promotes FGF signalling. Nat Cell Biol 2004, 6:38-44.

40. Fetchko M, Huang W, Li Y, Lai ZC: Drosophila Gp I 50 is required for early ommatidial development through modulation of Notch signaling. Embo J 2002, 2 I: I074-83.

4I. Ohta K, Lupo G, Kuriyama S, Keynes R, Holt CE, Harris WA, Tanaka $H$, Ohnuma S: Tsukushi functions as an organizer inducer by inhibition of BMP activity in cooperation with chordin. Dev Cell 2004, 7:347-58.

42. Beutler B, Jiang Z, Georgel P, Crozat K, Croker B, Rutschmann S, Du $X$, Hoebe K: Genetic analysis of host resistance: Toll-like receptor signaling and immunity at large. Annu Rev Immunol 2006, 24:353-89.

43. Ameye L, Young MF: Mice deficient in small leucine-rich proteoglycans: novel in vivo models for osteoporosis, osteoarthritis, Ehlers-Danlos syndrome, muscular dystrophy, and corneal diseases. Glycobiology 2002, I 2: I07R-I6R.

44. McGee AW, Yang Y, Fischer QS, Daw NW, Strittmatter SM: Experience-driven plasticity of visual cortex limited by myelin and Nogo receptor. Science 2005, 309:2222-6.

45. Long H, Sabatier C, Ma L, Plump A, Yuan W, Ornitz DM, Tamada A, Murakami F, Goodman CS, Tessier-Lavigne M: Conserved roles for Slit and Robo proteins in midline commissural axon guidance. Neuron 2004, 42:213-23.

46. Bando T, Sekine K, Kobayashi S, Watabe AM, Rump A, Tanaka M, Suda Y, Kato S, Morikawa Y, Manabe T, et al.: Neuronal leucinerich repeat protein 4 functions in hippocampus-dependent long-lasting memory. Mol Cell Biol 2005, 25:4I66-75.

47. Bermingham JR Jr, Shearin H, Pennington J, O'Moore J, Jaegle M, Driegen S, van Zon A, Darbas A, Ozkaynak E, Ryu El, et al.: The claw paw mutation reveals a role for Lgi4 in peripheral nerve development. Nat Neurosci 2006, 9:76-84.

48. Mi S, Miller RH, Lee X, Scott ML, Shulag-Morskaya S, Shao Z, Chan J, Thill G, Levesque $M$, Zhang $M$, et al.: LINGO-I negatively regulates myelination by oligodendrocytes. Nat Neurosci 2005 , 8:745-51

49. Matsushima N, Tachi N, Kuroki Y, Enkhbayar P, Osaki M, Kamiya M, Kretsinger $\mathrm{RH}$ : Structural analysis of leucine-rich-repeat variants in proteins associated with human diseases. Cell Mol Life Sci 2005, 62:2771-91.

50. Kalachikov S, Evgrafov O, Ross B, Winawer M, Barker-Cummings C, Martinelli Boneschi F, Choi C, Morozov P, Das K, Teplitskaya E, et al.: Mutations in LGII cause autosomal-dominant partial epilepsy with auditory features. Nat Genet 2002, 30:335-4I.

5I. Abelson JF, Kwan KY, O'Roak BJ, Baek DY, Stillman AA, Morgan TM, Mathews CA, Pauls DL, Rasin MR, Gunel M, et al.: Sequence variants in SLITRKI are associated with Tourette's syndrome. Science 2005, 3 1 0:3 17-20.

52. Bech-Hansen NT, Naylor MJ, Maybaum TA, Sparkes RL, Koop B, Birch DG, Bergen AA, Prinsen CF, Polomeno RC, Gal A, et al.: Mutations in NYX, encoding the leucine-rich proteoglycan nyctalopin, cause $X$-linked complete congenital stationary night blindness. Nat Genet 2000, 26:319-23.

53. Indo $Y$, Tsuruta M, Hayashida $Y$, Karim MA, Ohta K, Kawano T, Mitsubuchi $\mathrm{H}$, Tonoki $\mathrm{H}$, Awaya $\mathrm{Y}$, Matsuda I: Mutations in the TRKA/ NGF receptor gene in patients with congenital insensitivity to pain with anhidrosis. Nat Genet 1996, I3:485-8.

54. Majercak J, Ray WJ, Espeseth A, Simon A, Shi XP, Wolffe C, Getty K, Marine S, Stec E, Ferrer M, et al.: LRRTM3 promotes processing of amyloid-precursor protein by BACEI and is a positional candidate gene for late-onset Alzheimer's disease. Proc Nat Acad Sci USA 2006, I03: 17967-72.

55. Rougon G, Hobert $\mathrm{O}$ : New insights into the diversity and function of neuronal immunoglobulin superfamily molecules. Annu Rev Neurosci 2003, 26:207-38

56. Vogel C, Teichmann SA, Chothia C: The immunoglobulin superfamily in Drosophila melanogaster and Caenorhabditis elegans and the evolution of complexity. Development 2003, 130:6317-28.

57. Takeichi M: The cadherin superfamily in neuronal connections and interactions. Nat Rev Neurosci 2007, 8: I I-20.

58. Krogh A, Larsson B, von Heijne G, Sonnhammer EL: Predicting transmembrane protein topology with a hidden Markov model: application to complete genomes. I Mol Biol 200I, 305:567-80. 
59. Bendtsen JD, Nielsen H, von Heijne G, Brunak S: Improved prediction of signal peptides: SignalP 3.0. J Mol Biol 2004, 340:783-95.

60. Bateman A, Coin L, Durbin R, Finn RD, Hollich V, Griffiths-Jones S, Khanna A, Marshall M, Moxon S, Sonnhammer EL, et al:: The Pfam protein families database. Nucleic Acids Res 2004, 32:D I38-4I

61. Enright AJ, Van Dongen S, Ouzounis CA: An efficient algorithm for large-scale detection of protein families. Nucleic Acids Res 2002, 30:1575-84.

62. Kall L, Sonnhammer EL: Reliability of transmembrane predictions in whole-genome data. FEBS Lett 2002, 532:415-8.

63. Tusnady GE, Simon I: The HMMTOP transmembrane topology prediction server. Bioinformatics 200I, 17:849-50.

64. Hofmann K, Stoffel W: TMbase - A database of membrane spanning proteins segments. Biol Chem Hoppe-Seyler 1993, 374: 166

65. Eisenhaber B, Bork P, Eisenhaber F: Prediction of potential GPImodification sites in proprotein sequences. I Mol Biol 1999 292:74I-58

66. O'Connor E, Eisenhaber B, Dalley J, Wang T, Missen C, Bulleid N, Bishop PN, Trump D: Species specific membrane anchoring of nyctalopin, a small leucine-rich repeat protein. Hum Mol Genet 2005, I 4:1877-87.

67. Adachi-Yamada T, Harumoto T, Sakurai K, Ueda R, Saigo K, O'Connor MB, Nakato $\mathrm{H}$ : Wing-to-Leg homeosis by spineless causes apoptosis regulated by Fish-lips, a novel leucine-rich repeat transmembrane protein. Mol Cell Biol 2005, 25:3 I40-50.

68. Milan M, Perez L, Cohen SM: Boundary formation in the Drosophila wing: functional dissection of Capricious and Tartan. Dev Dyn 2005, 233:804-10.

69. Smits G, Kajava AV: LRRC8 extracellular domain is composed of 17 leucine-rich repeats. Mol Immunol 2004, 4 I:56 I-2.

70. Choe J, Kelker MS, Wilson IA: Crystal structure of human tolllike receptor 3 (TLR3) ectodomain. Science 2005, 309:58I-5.

71. Kim JI, Lee CJ, Jin MS, Lee CH, Paik SG, Lee H, Lee JO: Crystal structure of CD 14 and its implications for lipopolysaccharide signaling. J Biol Chem 2005, 280: I I347-5 I.

72. Huizinga EG, Tsuji S, Romijn RA, Schiphorst ME, de Groot PG, Sixma JJ, Gros P: Structures of glycoprotein Ibalpha and its complex with von Willebrand factor Al domain. Science 2002 297: II 76-9.

73. Fan QR, Hendrickson WA: Structure of human follicle-stimulating hormone in complex with its receptor. Nature 2005, 433:269-77.

74. McEwan PA, Scott PG, Bishop PN, Bella J: Structural correlations in the family of small leucine-rich repeat proteins and proteoglycans. J Struct Biol 2006, I 55:294-305.

75. Imamura F, Nagao H, Naritsuka H, Murata $Y$, Taniguchi H, Mori K: A leucine-rich repeat membrane protein, $5 T 4$, is expressed by a subtype of granule cells with dendritic arbors in specific strata of the mouse olfactory bulb. J Comp Neurol 2006, 495:754-68.

76. Nilsson J, Vallbo C, Guo D, Golovleva I, Hallberg B, Henriksson R, Hedman $\mathrm{H}$ : Cloning, characterization, and expression of human LIGI. Biochem Biophys Res Commun 200I, 284:I I55-6I.

77. Divanovic S, Trompette A, Atabani SF, Madan R, Golenbock DT, Visintin A, Finberg RW, Tarakhovsky A, Vogel SN, Belkaid Y, et al.: Negative regulation of Toll-like receptor 4 signaling by the Tolllike receptor homolog RP I05. Nat Immunol 2005, 6:57I-8.

78. Ollendorff $\mathrm{V}$, Noguchi T, deLapeyriere O, Birnbaum D: The GARP gene encodes a new member of the family of leucine-rich repeat-containing proteins. Cell Growth Differ 1994, 5:2 I 3-9.

79. Okazaki $Y$, Furuno M, Kasukawa T, Adachi J, Bono H, Kondo S, Nikaido I, Osato N, Saito R, Suzuki H, et al.: Analysis of the mouse transcriptome based on functional annotation of 60,770 fulllength cDNAs. Nature 2002, 420:563-73.

80. Davies AG, Spike CA, Shaw JE, Herman RK: Functional overlap between the mec-8 gene and five sym genes in Caenorhabditis elegans. Genetics 1999, 153:117-34.

8I. Matsushima N, Ohyanagi T, Tanaka T, Kretsinger RH: Super-motifs and evolution of tandem leucine-rich repeats within the small proteoglycans-biglycan, decorin, lumican, fibromodulin, PRELP, keratocan, osteoadherin, epiphycan, and osteoglycin. Proteins 2000, 38:210-25.

82. Tasheva ES, Klocke B, Conrad GW: Analysis of transcriptional regulation of the small leucine rich proteoglycans. Mol Vis 2004, 10:758-72.
83. Lein ES, Hawrylycz MJ, Ao N, Ayres M, Bensinger A, Bernard A, Boe AF, Boguski MS, Brockway KS, Byrnes EJ, et al:: Genome-wide atlas of gene expression in the adult mouse brain. Nature 2007, 445: $168-76$

84. Scott PG, McEwan PA, Dodd CM, Bergmann EM, Bishop PN, Bella J: Crystal structure of the dimeric protein core of decorin, the archetypal small leucine-rich repeat proteoglycan. Proc Natl Acad Sci USA 2004, 10 I: 15633-8.

85. Wang W, Yang Y, Li L, Shi Y: Synleurin, a novel leucine-rich repeat protein that increases the intensity of pleiotropic cytokine responses. Biochem Biophys Res Commun 2003, 305:98I-8.

86. Matthews KW, Mueller-Ortiz SL, Wetsel RA: Carboxypeptidase N: a pleiotropic regulator of inflammation. Mol Immunol 2004, 40:785-93.

87. Li CQ, Dong JF, Lanza F, Sanan DA, Sae-Tung G, Lopez JA: Expression of platelet glycoprotein (GP) $\mathbf{V}$ in heterologous cells and evidence for its association with GP Ib alpha in forming a GP Ib-IX-V complex on the cell surface. J Biol Chem 1995, 270:16302-7.

88. Satoh K, Hata M, Shimizu T, Yokota H, Akatsu H, Yamamoto T, Kosaka K, Yamada T: Lib, transcriptionally induced in senile plaque-associated astrocytes, promotes glial migration through extracellular matrix. Biochem Biophys Res Commun 2005, 335:63I-6.

89. Baxter RC: Insulin-like growth factor binding proteins in the human circulation: a review. Horm Res 1994, 42: 140-4

90. Roubin R, Pizette S, Ollendorff V, Planche J, Birnbaum D, Delapeyriere O: Structure and developmental expression of mouse Garp, a gene encoding a new leucine-rich repeat-containing protein. Int / Dev Biol 1996, 40:545-55.

9I. Jin H, Selfe J, Whitehouse C, Morris JR, Solomon E, Roberts RG: Structural evolution of the BRCAl genomic region in primates. Genomics 2004, 84: 107|-82.

92. [http://www.ncbi.n/m.nih.gov/FLC/getmgc.cgi]

93. Eddy SR: Profile hidden Markov models. Bioinformatics 1998 | 4:755-63.

94. HumanGPI: [http://mendel.imp.ac.at/gpi/Hs/hs.html]

95. FlyGPI: [http://mendel.imp.ac.at/gpi/Dm/dm.html].

96. Bell JK, Mullen GE, Leifer CA, Mazzoni A, Davies DR, Segal DM: Leucine-rich repeats and pathogen recognition in Toll-like receptors. Trends Immunol 2003, 24:528-33.

97. Gangloff M, Ludidi PL, Gay NJ: Structures and motifs involved in Toll signaling. In Toll receptors Edited by: Tina Rich. Eurekah.com and Kluwer Academic/Plenum Publishers; 2004.

Publish with Biomed Central and every scientist can read your work free of charge

"BioMed Central will be the most significant development for disseminating the results of biomedical research in our lifetime. "

Sir Paul Nurse, Cancer Research UK

Your research papers will be:

- available free of charge to the entire biomedical community

- peer reviewed and published immediately upon acceptance

- cited in PubMed and archived on PubMed Central

- yours - you keep the copyright
BioMedcentral 